\title{
Data-driven Robust Control of the Plasma Rotational Transform Profile and Normalized Beta Dynamics for Advanced Tokamak Scenarios in DIII-D
}

\author{
W. Shi ${ }^{\mathrm{a}}$, W.P. Wehner ${ }^{\mathrm{a}}$, J.E. Barton ${ }^{\mathrm{a}}$, M.D. Boyer ${ }^{\mathrm{a}}$, E. Schuster ${ }^{\mathrm{a}}$, \\ D. Moreau ${ }^{\text {b }}$, M.L. Walker ${ }^{\mathrm{c}}$, J.R. Ferron ${ }^{\mathrm{c}}$, T.C. Luce ${ }^{\mathrm{c}}$, D.A. Humphreys ${ }^{\mathrm{c}}$, \\ B.G. Penaflor ${ }^{\mathrm{c}}$, R.D. Johnson ${ }^{\mathrm{c}}$ \\ ${ }^{a}$ Mechanical Engineering and Mechanics, Lehigh University, Bethlehem, PA 18015, USA \\ ${ }^{b}$ CEA, IRFM, F-13018 St Paul lez Durance, France \\ ${ }^{c}$ General Atomics, San Diego, CA 92121, USA
}

\begin{abstract}
A control-oriented, two-timescale, linear, dynamic, response model of the rotational transform $l$ profile and the normalized beta $\beta_{N}$ is proposed based on experimental data from the DIII-D tokamak. Dedicated system-identification experiments without feedback control have been carried out to generate data for the development of this model. The data-driven dynamic model, which is both device-specific and scenario-specific, represents the response of the $\imath$ profile and $\beta_{N}$ to the electric field due to induction as well as to the heating and current drive (H\&CD) systems during the flat-top phase of an H-mode discharge in DIII-D. The control goal is to use both induction and the H\&CD systems to locally regulate the plasma $\imath$ profile and $\beta_{N}$ around particular target values close to the reference state used for system identification. A singular value decomposition (SVD) of the plasma model at steady state is carried out to decouple the system and identify the most relevant control channels. A mixed-sensitivity robust control design problem is formulated based on the dynamic model to synthesize a stabilizing feedback controller without input constraint that minimizes the reference tracking error and rejects external disturbances with minimal control energy. The feedback controller is then augmented with an anti-windup compensator, which keeps the given controller well-behaved in the presence of magnitude constraints in the actuators and leaves the nominal closed-loop system unmodified when no saturation
\end{abstract}

Email address: schuster@lehigh.edu (E. Schuster) 
is present. The proposed controller represents one of the first feedback profile controllers integrating magnetic and kinetic variables ever implemented and experimentally tested in DIII-D. The preliminary experimental results presented in this work, although limited in number and constrained by actuator problems and design limitations, as it will be reported, show good progress towards routine current profile control in DIII-D and leave valuable lessons for further advancements in the field.

Keywords:

Plasma Control, Advanced Scenarios Control, Data-driven Model-based Control, Safety Factor Profile Control

\section{Introduction}

The planned ITER reactor [1] will be capable of exploring advanced tokamak (AT) modes of operation, characterized by high plasma pressure, long confinement times, and low levels of inductively driven plasma current, which allow for steady-state operation. These advanced modes require active feedback control to maintain and develop high performance plasmas, good confinement, and long plasma discharges. In particular, the control of the plasma current profile, which is intimately related to the safety factor profile $q$, or its inverse, the rotational transform $l$ profile, is critical for the development and sustainment of the selfgenerated, non-inductive, bootstrap current, which in turn serves as an enabler for steady-state operation. Early experiments in different tokamaks (Tore Supra [2, 3], JT-60U [4, 5], DIII-D [6]) have demonstrated feasibility of achieving current profile control. Most of this prior work has focused, however, on non-model-based control of scalar parameters characterizing the current profile such as the internal inductance $l_{i}$, the safety factor at magnetic axis $q_{0}$, or the minimum value of the safety factor $q_{\min }$. While the control of scalar parameters such as $q_{\min }$ is critical to mitigate plasma instabilities and improve confinement, the shaping of the entire $q$ profile is necessary to maximize the fraction of bootstrap current and maintain stability in advanced scenarios. Therefore, techniques to actively control the evolution of the full $q$ profile in closed loop during the discharge are of paramount importance to the realization of advanced scenarios in ITER. The high dimensionality of this problem, along with the strong coupling between magnetic and kinetic profiles, motivate the use of model-based control synthesis that can accommodate this complexity by embedding physics-based dynamic models within the design. 
Mathematical modeling of plasma transport phenomena with sufficient complexity to capture the dominant dynamics is therefore critical for plasma profile control design. Transport theories, even under restrictive assumptions, produce strongly nonlinear models based on partial differential equations (PDEs). The complexity of these first-principles models needs to be reduced to facilitate design of compact and reliable control strategies. During this control-oriented model reduction process $[7,8,9]$, there is always a trade-off between the simplicity of the model and both its physics accuracy and its range of validity. First-principlesdriven modeling provides the freedom of arbitrarily handling this trade-off and deciding on the level of simplicity, accuracy and validity of the model. This features allows, for instance, for the derivation of models capturing the nonlinear response of the current profile to control actuation. First-principles-driven controloriented models have been recently used to determine optimal open-loop (feedforward) actuator trajectories that achieve and sustain a desired $q$ profile $[10,11,12]$, and to design complementing closed-loop (feedback) control laws that add robustness against disturbances and model uncertainties. Several approaches have been recently proposed for first-principles-driven current profile feedback control $[13,14,15,16,17,18,19]$ and some of them have been experimentally tested in DIII-D [20, 21, 22]. Data-driven modeling techniques such as linear system identification [23] emerge as an alternative to first-principles-driven modeling and have the potential to obtain low-complexity dynamic models. This modeling approach lacks however the ability of arbitrarily deciding on the level of simplicity, accuracy and validity of the model since it directly produces linear models based on ordinary differential equations (ODEs). As the identified models are linear, they are only valid around the reference plasma state adopted during the system identification experiment. Therefore, the effectiveness of the controllers synthesized based on these models may be limited when the plasma state moves away from the reference state. Moreover, as these models are device-specific, dedicated system identification experiments are needed in each device, and potentially for each control scenario, to develop model-based controllers. In spite of these limitations, data-driven control-oriented models can be useful for the design of local regulators around the reference state. In the JET tokamak, a two-timescale linear model has been identified from data to describe the dynamic behavior of the magnetic and kinetic profiles around certain quasi-steady-state trajectories, and a feedback controller has been designed based on two loops operating on the magnetic and kinetic time scales respectively $[24,25]$. Input/output (I/O) diagnostic data has also been generated in dedicated system identification experiments on JT-60U and DIII-D [26] to identify magnetic and kinetic profile response models. 
In this work, a two-timescale dynamic model of the rotational transform $\imath$ profile and the normalized beta $\beta_{N}$ is identified from DIII-D data. The normalized beta $\beta_{N}$ is a key measure of performance used to gauge progress toward developing a power-producing fusion reactor and is closely related to MHD stability, which makes real-time control of the $\imath$ profile and $\beta_{N}$ of paramount importance. A model-based, multi-input-multi-output (MIMO) controller is synthesized based on the identified model for the simultaneous regulation of the $\imath$ profile and $\beta_{N}$ during the current flat-top phase in $\mathrm{H}$-mode discharges. A singular value decomposition (SVD) is used to decouple the combined system and identify the most relevant control channels. The mixed-sensitivity robust control method $[27,28]$ is applied to synthesize a closed-loop controller that minimizes the reference tracking error and rejects external disturbances with minimal control energy. The feedback controller is then augmented with an anti-windup compensator [29, 30], which keeps the given $\imath$ profile and $\beta_{N}$ controller well-behaved in the presence of actuator constraints and leaves the nominal closed-loop system unmodified when no saturation in the actuators is present. The proposed controller has been tested both in simulations and in a limited number of experiments on the DIII-D tokamak. These experiments, together with those reported in [31, 32, 33, 34], mark the first time ever data-driven, model-based, current-profile controllers were implemented and tested in DIII-D. The experimental results, which are thoroughly analyzed in this work, are partially successful due to actuator constraints and design limitations. Nevertheless, these preliminary results represent an incremental step towards routine current profile control in DIII-D and provide valuable lessons regarding model identification, control design and controllability that will be exploited to achieve this goal.

This paper is organized as follows. In Section 2, the system identification procedure carried out in the DIII-D tokamak is briefly described, and the dynamic linear models relating the rotational transform $\imath$ profile and $\beta_{N}$ to the plasma current $\left(I_{p}\right)$, neutral beam injection (NBI), and electron cyclotron (EC) heating and current drive $(\mathrm{H} \& \mathrm{CD})$ are developed. In Section 3, the design of the control algorithm and the anti-windup compensator is introduced. Simulated and experimental results in DIII-D illustrating the performance of the controllers are presented in Section 4. Conclusions and future research directions are stated in Section 5. 


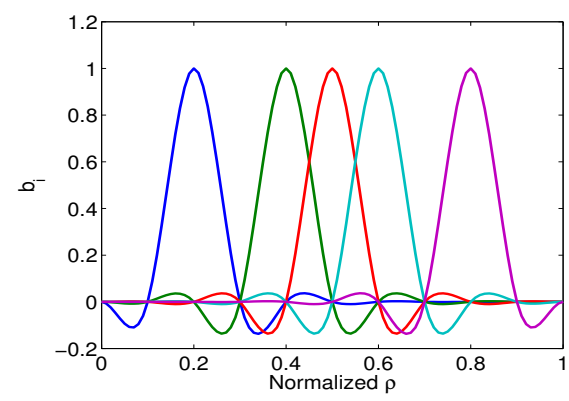

Figure 1: Cubic splines, $b_{i}$, used for the expansion of the $\imath$ profile.

\section{System Identification on DIII-D}

In order to use multivariable control theory for the synthesis of a controller capable of regulating the plasma $l$-profile and $\beta_{N}$ evolutions in advanced tokamak scenarios, system identification techniques are used to develop from measured data a control-oriented dynamic model for the linear response of these variables to the actuators. A Galerkin scheme [35] is used to convert the infinite-dimensional (PDE) transport equation for the $l$-profile to a finite-dimensional (ODE) model in space. The Galerkin scheme assumes that by increasing the number of basis functions the identified ODE model will converge towards the PDE that best fits the data. The $\imath$ profile in normalized radial coordinates is then approximated by the sum of a discrete number of spatial functions,

$$
\imath(\hat{\rho}, t) \approx \sum_{i=1}^{N} G_{i}(t) b_{i}(\hat{\rho})
$$

where the expansion coefficients $G_{i}(t), i=1, \ldots, N$, are called Galerkin coefficients, and the spatial functions $b_{i}(\hat{\rho}), i=1, \ldots, N$, are called Galerkin basis functions. In this work, system identification for the plasma rotational transform profile $l(\hat{\rho}, t)$ is carried out based on five cubic-splines Galerkin basis functions centered around normalized radii $\hat{\rho}=0.2,0.4,0.5,0.6,0.8$ as shown in Fig. 1. The choice of these spatial coordinates was motivated by the interest in obtaining a low-order finite-dimensional representation of the whole profile dynamics, which led to the selection of just five points evenly distributed around the profile midpoint at $\hat{\rho}=0.5$. The parameter $\hat{\rho}$ is the normalized effective minor radius, which can be denoted as $\hat{\rho}=\frac{\rho}{\rho_{b}}$, where $\rho$ is the mean effective minor radius of the flux surface, i.e., $\pi B_{\phi, 0} \rho^{2}=\Phi$. The parameter $\Phi$ is the toroidal magnetic flux, and $B_{\phi, 0}$ is the toroidal magnetic field at the geometric major radius. The parameter $\rho_{b}$ is the mean effective minor radius of the last closed magnetic flux surface. 
To collect data for system identification a number of discharges were run with identical ramp-up phases and different flat-top phases characterized by varied actuator modulations schemes that excited the system around a predefined reference state. The reference plasma state was that of a $1.8 \mathrm{~T}, \beta_{N^{-}}$-controlled AT scenario, with a center plasma density $n_{e o} \approx 5 \times 10^{19} \mathrm{~m}^{-3}$ and plasma current $I_{p}=0.9 \mathrm{MA}$. The scenario was developed to achieve non-inductive current fractions near unity, bootstrap current fractions larger than $65 \%$, and a normalized confinement factor $H_{98(y, 2)} \approx 1.5$ [26]. Actuator modulations were applied from $t=2.5 \mathrm{~s}$, and the $I_{p}$ and $\beta_{N}$ controls were disabled to ensure no feedback response during data collection. The EC and NBI systems provided the non-inductive heating and current drive sources for these experiments. The EC system in DIII-D is composed by six gyrotrons with individual nominal power of around $1 \mathrm{MW}$ at a central frequency of $110 \mathrm{GHz}$. The NBI system in DIII-D consists of four beam-lines, with each beam-line having two ion sources in parallel. Each ion source produces an 80 $\mathrm{keV}$ deuterium beam and can inject a maximum of $2.5 \mathrm{MW}$ of power into the plasma. Three of the beam-lines $\left(30^{\circ}, 150^{\circ}, 330^{\circ}\right)$ inject power in the same direction as the usual plasma current. The $210^{\circ}$ system is used for counter-injection, since its beam is injected in the opposite direction. Each beamline is aligned at an angle $19.5^{\circ}$ from the radial through the injection port. Available beam-lines and gyrotrons were grouped to form, together with $I_{p}$, five independent H\&CD actuators: ( $i$ ) plasma current $I_{p},(i i)$ co-current NBI power $P_{C O}(150 \mathrm{~L}$ and $330 \mathrm{~L})$, (iii) counter-current NBI power $P_{C T}(210 \mathrm{R})$, (iv) balanced NBI power $P_{B L}(150 \mathrm{R}$ and $210 \mathrm{~L}$ ), and (v) total EC power from all gyrotrons $P_{E C}$. All actuators were modulated individually in open loop while the other actuators were held at their respective reference values. Several shots (\#140076, 140077, 140093, 140106, and 140107) were used to identify the model. Shot 140107 performs modulation of $I_{p}$, shots 140076, 140077, and 140106 perform modulation of the neutral beam groups, and shot 140093 performs modulation of the total EC power. Typical $\imath$ profile, $q$ profile, $I_{p}$ and $\beta_{N}$ at the beginning of the system identification experiments is given by shot \#140090 in Fig. 8 and Table 3. More details on the system identification experiments can be obtained from our previous work [26, 36].

The relation between inputs and outputs for any shot can be assumed in the form of

$$
y(t)=y_{F F}+\Delta y(t)=P_{F F}\left(u_{F F}\right)+P \Delta u(t),
$$

where $P_{F F}$ represents the relationship between the reference (feedforward) input $u_{F F}$ and the reference (feedforward) output $y_{F F}$. The variable $\Delta y(t)$ denotes the deviation output defined as $\Delta y(t)=\left[\Delta \imath(t), \Delta \beta_{N}(t)\right]=y(t)-y_{F F}$, with $y(t)=$

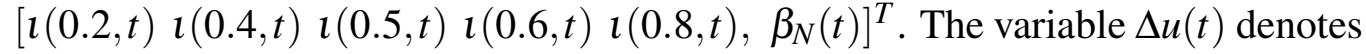


the deviation input defined as $\Delta u=u-u_{F F}$ with $u=\left[I_{p}, P_{C O}, P_{C T}, P_{B L}, P_{E C}\right]$. By subtracting the feedforward value from our data set, we only consider the linear dynamics $\Delta y(t)=P \Delta u(t)$. The linear model $P$ is identified from experimental data using the prediction error method (PEM) according to a least squares fit criterion [23]. By using a finite-dimensional approximation of the $\imath$ profile as proposed in (1) and neglecting the nonlinear dynamics, the structure of the to-be-identified model $P$ is derived from the transport equations and written as

$$
\begin{gathered}
\Delta i(t)=A_{11} \Delta l(t)+A_{12} \Delta \beta_{N}(t)+B_{1} \Delta u(t) \\
\varepsilon \Delta \dot{\beta}_{N}(t)=A_{21} \Delta l(t)+A_{22} \Delta \beta_{N}(t)+B_{2} \Delta u(t),
\end{gathered}
$$

where the parameter $\varepsilon<<1$, representing the typical ratio between the energy confinement time and the characteristic resistive diffusion time, is used to indicate the existence of two timescales in the system. The magnetic variable is characterized by a slow dynamics with timescale given by $\tau_{m a g}=t$ and the kinetic variable is characterized by a fast dynamics with timescale given by $\tau_{k i n}=t / \varepsilon$. The study of dynamic systems with two clear timescales has a long history in nonlinear control analysis, which is summarized by the theory of singular perturbations [37]. The modeling of the current profile dynamics as a two-timescale system indeed simplifies the associated control design. Therefore, the natural timescale separation observed in the system has been exploited in the past at the moment of developing a control-oriented model following both first-principles-driven [7] and data-driven [25] approaches. Following a similar approach in this work allows us to rewrite the model (3)-(4) as the combination of a slow model

$$
\Delta i(t)=A_{s} \Delta l(t)+B_{s} \Delta u_{s}(t) \quad \Delta \beta_{N s}(t)=C_{s} \Delta l(t)+D_{s} \Delta u_{s}(t),
$$

and a fast model

$$
\Delta \dot{\beta}_{N f}(t)=A_{f} \Delta \beta_{N f}(t)+B_{f} \Delta u_{f}(t),
$$

where $\Delta \beta_{N s}$ and $\Delta \beta_{N f}$ are the slow and fast components of $\Delta \beta_{N}$, and $\Delta u_{s}$ and $\Delta u_{f}$ are the slow and fast components of $\Delta u$. It is straightforward to show that the matrices to be identified in (5)-(6) are linked to the original model matrices in (3)-(4) through the relations:

$$
\begin{array}{lll}
A_{s}=A_{11}-A_{12} A_{22}^{-1} A_{21}, & C_{s}=-A_{22}^{-1} A_{21}, & A_{f}=\varepsilon^{-1} A_{22}, \\
B_{s}=B_{1}-A_{12} A_{22}^{-1} B_{2}, & D_{s}=-A_{22}^{-1} B_{2}, & B_{f}=\varepsilon^{-1} B_{2} .
\end{array}
$$

Because $\varepsilon<<1$ (denoting the natural timescale separation in the system), we usually have $A_{21} / \varepsilon, A_{22} / \varepsilon>>A_{11}, A_{12}$ and $B_{2} / \varepsilon>>B_{1}$. Therefore, identifying the system matrices in (5)-(6) instead of the system matrices in (3)-(4) 
avoids the risk of having seriously ill-conditioned system matrices and making the synthesis of a reliable control strategy even more challenging. Rewriting the model (3)-(4) as (5)-(6) requires the assumption that the $l$ profile dynamics has a limited bandwidth, which implies that its response to the control input $\Delta u$ is identical to its response to the low-frequency-content control input $\Delta u_{s}$ since the high-frequency-content control input $\Delta u_{f}$ is filtered by the system itself. Stating the two-timescale model structure as in equations (5)-(6) leaves the designer with another design choice to make, which is the value of the cutoff frequency separating the slow (low-frequency) content and the fast (high-frequency) content of the control input. The cutoff frequency selection may become particularly challenging when the plasma current is used as a control input, as in this work, since in this case the outer part of the $\imath$ profile close to the plasma boundary (e.g., $l(0.8, t)$ in this work) may respond relatively fast to changes in the plasma current. Selecting a cutoff frequency too low in the effort of maximizing the separation between slow and fast dynamics may indeed be restrictive and may pose a risk to closedloop performance due to the neglected dynamics (the response of the $l$ profile to the low-frequency-content control input $\Delta u_{s}$ may be largely different from its response to the control input $\Delta u$, violating the model assumption). In this work, the lowest frequency at which the inputs used in the system identification experiments $[26,36]$ could be filtered while retaining a good fit of the unfiltered $\imath$ data by the slow model has been found to be around $1 \mathrm{~Hz}$. Therefore, the filter cutoff frequency has been chosen as $1 \mathrm{~Hz}$ to separate the slow and fast components of the input $\Delta u$ and $\Delta \beta_{N}$. It is important to note, however, that this value of the cutoff frequency is indeed related to the frequency content of the excitation (input) signals during the system identification experiments. If higher-frequency excitation signals had been used, a higher value for the cutoff frequency would have been most likely necessary. The presence of higher-frequency excitation signals in the closed-loop experiments could make the chosen cutoff frequency of $1 \mathrm{~Hz}$ too low and in part responsible for some closed-loop performance limitations observed in the experiments as it will be discussed later in the paper. As stated above, a cutoff frequency that is too low violates the model assumption, neglects important $l$-profile dynamics, and therefore affects the performance of the model-based controller.

A model validation procedure has been carried out by comparing the model prediction with experimental data from shots not used in the system identification procedure. This comparison includes both $l$ at $\hat{\rho}=0.2,0.4,0.6,0.8$ and $\beta_{N}$. Fig. 2 illustrates the cases of shots \#140094 and \#140109 as examples. While Fig. 2 (a) and (c) display the experimental inputs, Fig. 2 (b) and (d) compare predictions 

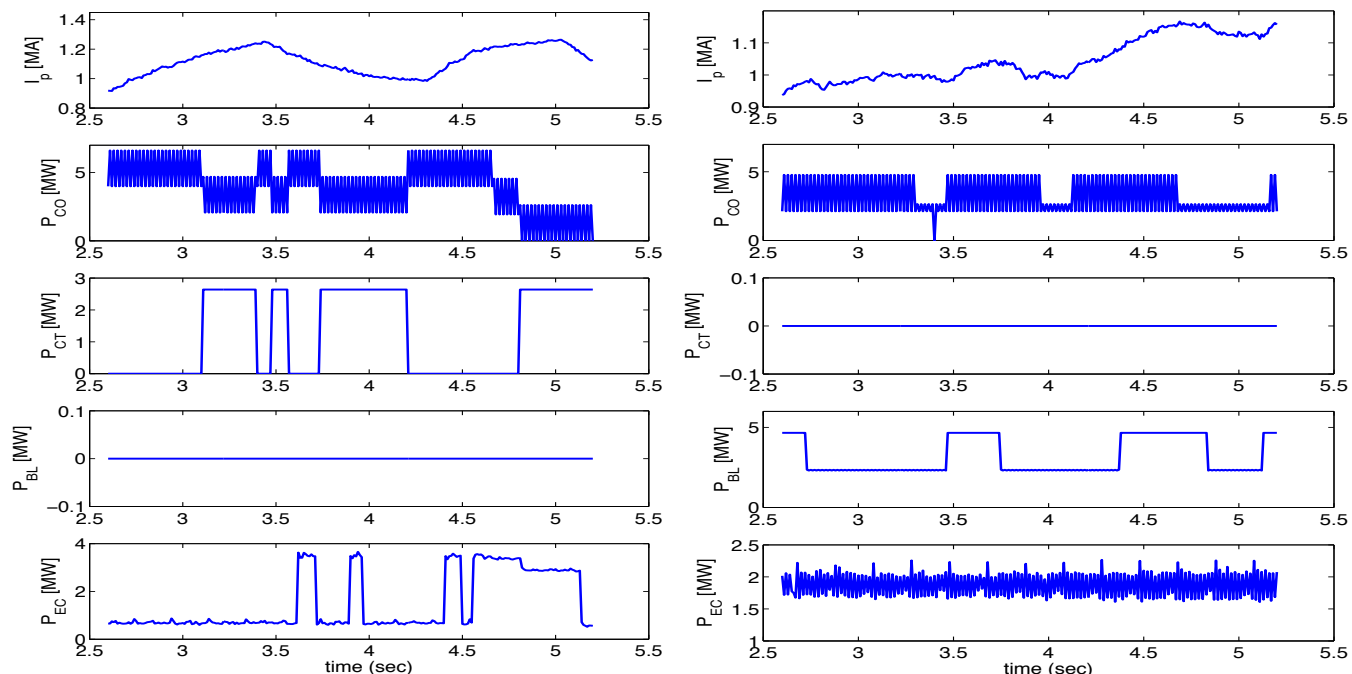

(a)
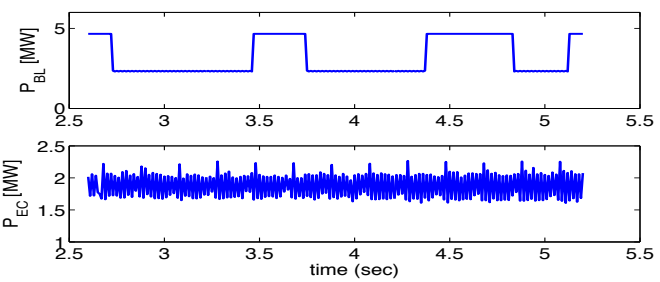

(b)
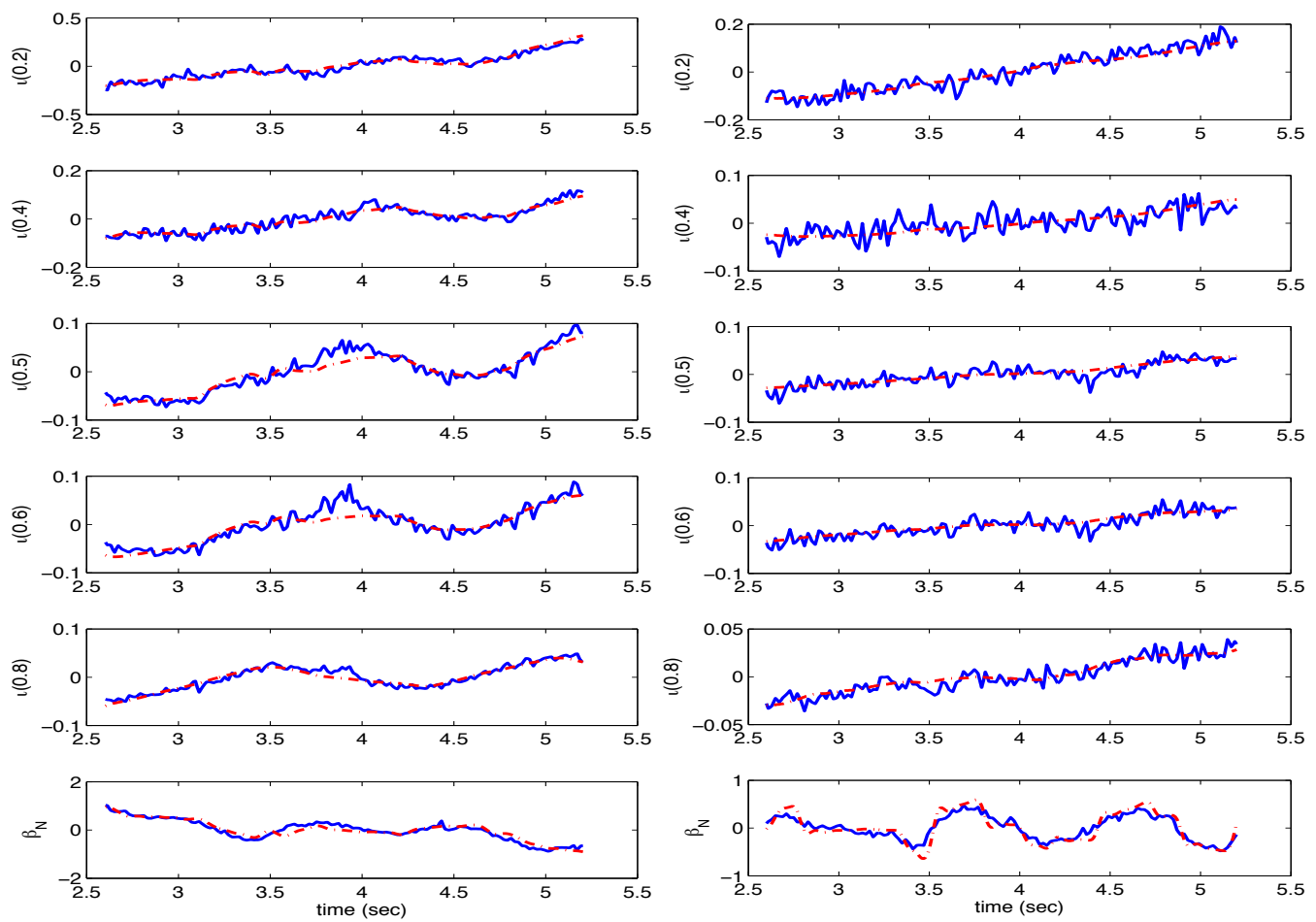

(c)

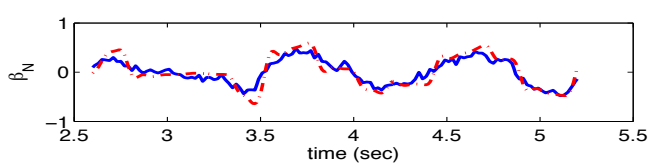

(d)

Figure 2: Experimental actuator modulation: (a) Shot 140094; (b) Shot 140109. Comparison between experimentally measured (blue solid line) and model-predicted (red dashed-dotted line) $\imath$ profile and $\beta_{N}$ : (c) Shot 140094 (fit functions (top to bottom): $f^{1}=73.33 \%, f^{2}=68.85 \%$, $f^{3}=67.87 \%, f^{4}=58.80 \%, f^{5}=71.32 \%, f^{6}=66.56 \%$ ) ; (d) Shot 140109 (fit functions (top to bottom): $f^{1}=71.98 \%, f^{2}=29.78 \%, f^{3}=50.12 \%, f^{4}=53.00 \%, f^{5}=65.75 \%, f^{6}=49.17 \%$ ). 
with experimental data. Fit functions $f^{i}$ are introduced to quantify the relationship between the measured data $y_{m}^{i}$ and the model-predicted data $y^{i}$, where the superindex denotes the $i$-th channel of the system output, i.e.,

$$
f^{i}=1-\left(\sum_{k=1}^{N}\left[y_{m}^{i}\left(t_{k}\right)-y^{i}\left(t_{k}\right)\right]^{2} / \sum_{k=1}^{N}\left[y_{m}^{i}\left(t_{k}\right)-<y_{m}^{i}>\right]^{2}\right)^{1 / 2},
$$

where $f^{i}=1$ is a perfect fit and $f^{i}=0$ corresponds to a reconstructed data set identically equal to the mean of the measured data, $\left\langle y_{m}^{i}\right\rangle$. Because the noise is included in the measured data from the experiment, the values of the fit functions are not sought to be 1 since that would mean that the identified model fits the noise perfectly, which is undesired. A "good" value, based on the definition of the fit functions, will therefore depend on the noise level present in the system and the confidence bands assumed by the designer. Fig. 2 (b) and (d) display the typical fit between measured and model-predicted data observed in the model validation procedure, which involved a larger number of shots and consistently showed the type of qualitative agreement expected from a control-oriented model. Note that although the fit function values (provided in the figure caption) are far from 1, the control-oriented model seems to capture the trend of the system in response to the control actuation, which is the objective of the system identification procedure (not the perfect fit between predicted and experimental data). It is often not possible, however, to assess the true requirements for model accuracy until experimental tests of the model-based controller are performed. Therefore, an iterative process may be necessary.

\section{Control System Design}

\subsection{Control System Structure}

A multi-input-multi-output (MIMO) robust feedback controller based on the identified linear models (5) and (6) are proposed for the regulation of the evolution of the $\imath$ profile and $\beta_{N}$ on DIII-D. In order to cope with the limitations on achievable values and rates for the actuators (plasma current, beam powers, gyrotron powers) we follow an a posteriori design approach where the MIMO robust feedback controller is modified by an anti-windup compensator. The control design procedure is summarized by the following steps: (i) decouple the system and identify the most relevant control channels, (ii) design an $H_{\infty}$ controller $\hat{K}$ ignoring control input saturation, (iii) add an anti-windup compensator $A W$ to minimize the adverse effect of any control input saturation on the closed loop performance. The overall control system including the MIMO $H_{\infty}$ controller and the anti-windup 


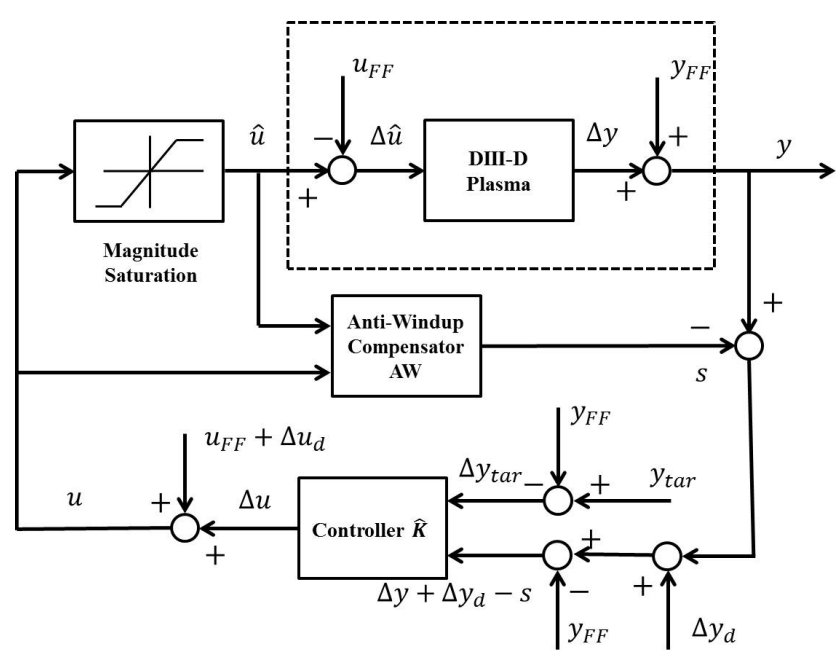

Figure 3: DIII-D 1 -profile $+\beta_{N}$ control system architecture.

compensator is shown in Fig. 3. By subtracting the reference (feedforward) output values $y_{F F}$ from the measured output values $y$, the deviation output $\Delta y=y-y_{F F}$ is generated and used as the input to the feedback controller. The objective of the feedback controller is to make the deviation output $\Delta y=y-y_{F F}$ follow the target output $\Delta y_{t a r}=y_{t a r}-y_{F F}$ under the influence of input $\left(\Delta u_{d}\right)$ and output $\left(\Delta y_{d}\right)$ disturbances, i.e., to drive the tracking error $e(t)=\Delta y_{\text {tar }}(t)-\Delta y(t)$ close to zero. The target output $y_{\text {tar }}$ represents the desired values for $\beta_{N}$ and the $\imath$ profile at $\hat{\rho}=0.2,0.4,0.5,0.6,0.8$. The output of the feedback controller $\Delta u$ is added to the reference (feedforward) input values $u_{F F}$ to generate the overall input values $u$ requested to the actuators. The differences between requested $(u)$ and achieved $(\hat{u})$ input values drive the anti-windup compensator that eventually modifies the measured output values $y$ through the signal $s$ when any of the actuators saturate.

\subsection{Decoupling and Identification of Most Relevant Control Channels}

The relation between the inputs and the outputs of the linear models (5) and (6) can be expressed in terms of its transfer function $P(s)$, i.e.,

$$
\frac{\Delta Y(s)}{\Delta U(s)}=P(s)=C(s I-A)^{-1} B
$$

where $s$ denotes the Laplace variable and $\Delta Y(s)$ and $\Delta U(s)$ denote the Laplace transforms of the output $\Delta y$ and the input $\Delta u$ respectively. Assuming a constant 

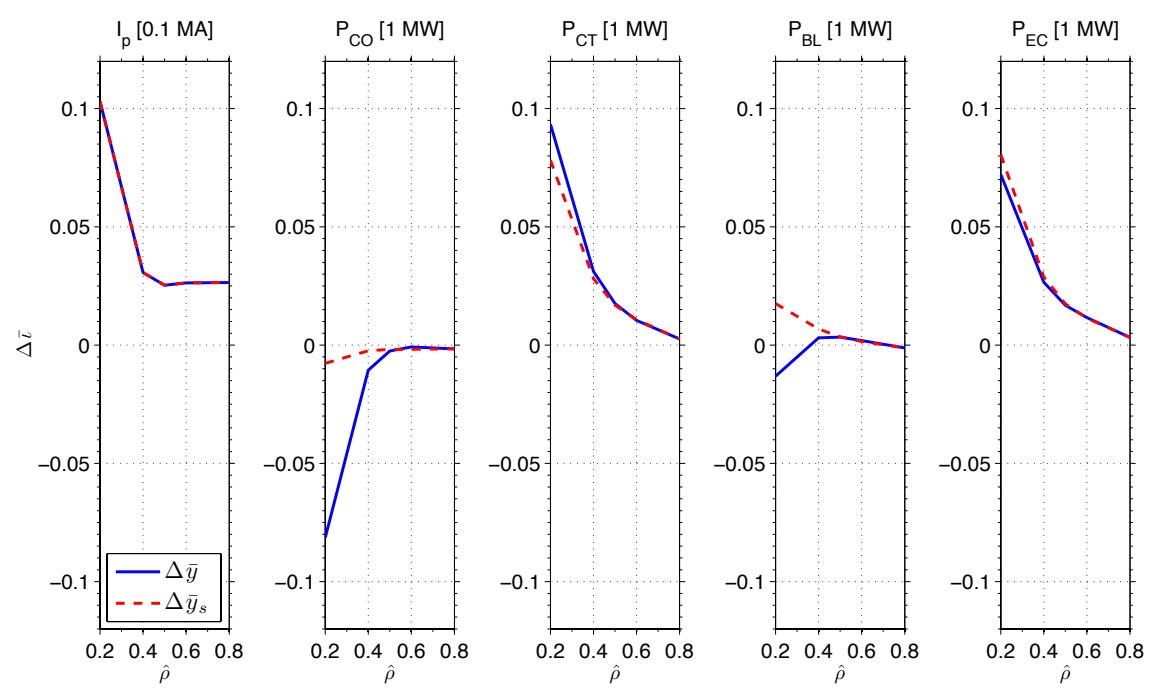

Figure 4: Steady-state gains for the $l$-profile response. The steady-state output $\Delta \bar{y}$ (solid blue) is compared with the significant steady-state output $\Delta \bar{y}_{s}$ (dashed red) for $k=2$. The powers are expressed in MW and the current in units of 0.1 MA. The weight matrices are chosen in this case as $R=\operatorname{diag}([0.01,0.25,1000,0.5,0.25])$ and $Q=\operatorname{diag}([1,1,1,1,1,1])$.

target $\Delta \bar{y}_{\text {tar }}$ and closed-loop stabilization, the system will reach steady state as $t \rightarrow \infty$. It is possible to define

$$
\Delta \bar{y}=\lim _{t \rightarrow \infty} \Delta y(t), \quad \Delta \bar{u}=\lim _{t \rightarrow \infty} \Delta u(t), \quad \bar{e}=\lim _{t \rightarrow \infty} e(t)=\Delta \bar{y}_{t a r}-\Delta \bar{y},
$$

where $e=\Delta y_{\text {tar }}-\Delta y$ denotes the tracking error. Under these assumptions, the closed-loop system is specified by

$$
\begin{aligned}
& \Delta \bar{y}=\bar{P} \Delta \bar{u}=-C A^{-1} B \Delta \bar{u}, \\
& \Delta \bar{u}=\overline{\hat{K}} \bar{e}=\overline{\hat{K}}\left(\Delta \bar{y}_{t a r}-\Delta \bar{y}\right),
\end{aligned}
$$

where $\bar{P}=P(0)=-C A^{-1} B+D$ is the steady state transfer function, $\hat{K}(s)$ is the transfer function of the to-be-designed controller and $\overline{\hat{K}}=\hat{K}(0)$. The steady state gains of the identified model for the $\imath$ profile are shown in Fig. 4, where the steadystate response $\Delta \bar{l}$ to unitary changes in the various inputs is plotted. The plasma current is the most capable actuator in adjusting the $l$ profile in absolute terms. The co-injection and counter-injection beams are also very powerful, affecting the profile in different directions in agreement with prior experiments. The EC power leads to an increase in the $\Delta l$ profile, and the balanced-injection beams nearly do not have influences in the $\imath$ profile. 
Singular value decomposition (SVD) is employed to decouple the system and determine the most significant input-output channels for tracking based on the steady state transfer function $\bar{P}$. Symmetric positive definite matrices $Q \in \mathfrak{R}^{p \times p}$, where $p=6$ is the number of outputs, and $R \in \mathfrak{R}^{m \times m}$, where $m=5$ is the number of inputs, are introduced to weigh the tracking error and control effort, respectively. The singular value decomposition of the "weighted" steady-state transfer function is given by

$$
\tilde{P}=Q^{1 / 2} \bar{P} R^{-1 / 2}=U S V^{T},
$$

where $S=\operatorname{diag}\left(\sigma_{1}, \sigma_{2}, \cdots, \sigma_{m}\right) \in \mathfrak{R}^{m \times m}$ since $m<p, U \in \mathfrak{R}^{p \times m}, V \in \mathfrak{R}^{m \times m}$. The matrices $U$ and $V$ are unitary, i.e., $U^{T} U=I, V^{T} V=V V^{T}=I$. The steady-state input-output relation can now be expressed as

$$
\Delta \bar{y}=Q^{-1 / 2} \tilde{P} R^{1 / 2} \Delta \bar{u}=Q^{-1 / 2} U S V^{T} R^{1 / 2} \Delta \bar{u} .
$$

By invoking the properties of the SVD, the columns of the matrix $Q^{-1 / 2} U S$ define a basis for the subspace of obtainable steady-state output values. Therefore, it is always possible to write

$$
\Delta \bar{y}=Q^{-1 / 2} U S \Delta \bar{y}^{*} \Longleftrightarrow \Delta \bar{y}^{*}=S^{-1} U^{T} Q^{1 / 2} \Delta \bar{y},
$$

where $\Delta \bar{y}^{*} \in \mathfrak{R}^{m}$ denotes the basis coefficients of the component of the output signal that is indeed achievable. This implies that only the component of the reference vector $\Delta \bar{y}_{\text {tar }}$ that lies in this subspace will be trackable. The reference vector is now written as the sum of a trackable component $\Delta \bar{y}_{\text {tar }}$ and a non-trackable component $\Delta \bar{y}_{t a r_{n t}}$, i.e., $\Delta \bar{y}_{t a r}=\Delta \bar{y}_{t a r_{t}}+\Delta \bar{y}_{t a r_{n t}}$, where

$$
\Delta \bar{y}_{\text {tar }_{t}}=Q^{-1 / 2} U S \Delta \bar{y}_{\text {tar }}^{*} \Longleftrightarrow \Delta \bar{y}_{\text {tar }}^{*}=S^{-1} U^{T} Q^{1 / 2} \Delta \bar{y}_{t a r},
$$

with $\Delta \bar{y}_{\text {tar }}^{*} \in \mathfrak{R}^{m}$ representing the basis coefficients of the component of the target signal that is indeed trackable and $S^{-1} U^{T} Q^{1 / 2} \Delta \bar{y}_{t a r_{n t}}=0$. By defining

$$
\Delta \bar{u}^{*}=V^{T} R^{1 / 2} \Delta \bar{u}
$$

where $\Delta \bar{u}^{*} \in \mathfrak{R}^{m}$, the relationship between $\Delta \bar{y}^{*}$ and $\Delta \bar{u}^{*}$ is obtained by using (13), (14), (16) as

$$
\Delta \bar{y}^{*}=S^{-1} U^{T} Q^{1 / 2} \Delta \bar{y}=S^{-1} U^{T} Q^{1 / 2} Q^{-1 / 2} U S V^{T} R^{1 / 2} \Delta \bar{u}=\Delta \bar{u}^{*} .
$$

This defines a one-to-one relationship between the transformed inputs and outputs, i.e., $\Delta \bar{y}^{*}=\Delta \bar{u}^{*}$, which leads to a square and decoupled system. 


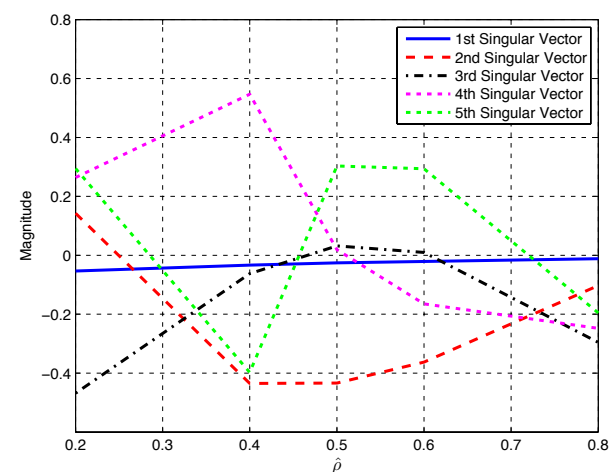

(a)

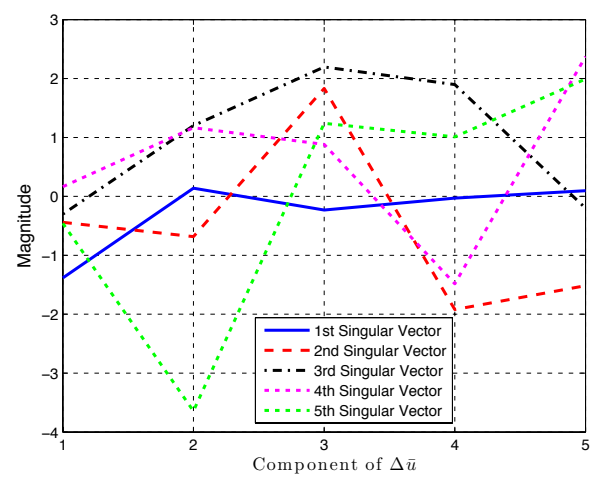

(b)

Figure 5: (a) Output singular vectors $Q^{-1 / 2} U$, (b) Input singular vectors $V^{T} R^{1 / 2}$ from the SVD analysis $\left(\sigma_{1}=106.2544, \sigma_{2}=0.2697, \sigma_{3}=0.0624, \sigma_{4}=0.0077\right.$, and $\left.\sigma_{5}=0.0009\right)$.

The bases obtained through the singular value decomposition of the steadystate response predicted by the model are shown in Fig. 5. The singular output vectors given by the columns of $Q^{-1 / 2} U$, which define the subspace of obtainable steady state output values and therefore the trackable component of the reference vector, are shown in Fig. 5 (a). The corresponding input singular vectors given by the rows of $V^{T} R^{1 / 2}$, which define the associated steady-state input values, are shown in Fig. 5 (b). As evidenced by the magnitude of the first singular value relative to the others, the first output singular vector is the dominant shape of an achievable steady state profile according to the model. In order to generate this profile shape, the feedback controller must actuate in the direction associated with the first input singular vector. As the value of the singular value decreases, a larger amount of control effort is needed along the direction of the associated input singular vector to produce a significant contribution to the steady state profile in the direction of the associated output singular vector.

To quantify the tracking performance in steady state, a performance index can be defined as

$$
\bar{J}=\lim _{t \rightarrow \infty} e^{T}(t) Q e(t)=\bar{e}^{T} Q \bar{e},
$$

where the steady-state tracking error $\bar{e}$ can now be rewritten as

$$
\bar{e}=\Delta \bar{y}_{t a r}-\Delta \bar{y}=Q^{-1 / 2} U S\left(\Delta \bar{y}_{t a r}^{*}-\Delta \bar{y}^{*}\right) .
$$


By substituting this expression into (18), the performance index is expressed as

$$
\bar{J}=\left(\Delta \bar{y}_{\text {tar }}^{*}-\Delta \bar{y}^{*}\right)^{T} S^{2}\left(\Delta \bar{y}_{\text {tar }}^{*}-\Delta \bar{y}^{*}\right)=\sum_{i=1}^{m} \sigma_{i}^{2}\left(\Delta \bar{y}_{\text {tar }}^{*}-\Delta \bar{y}_{i}^{*}\right)^{2} .
$$

The goal of the controller to be introduced in the next subsection is to minimize the tracking error by driving $\Delta y_{i}^{*}$ towards $\Delta y_{t a r_{i}}^{*}$, for $i=1, \ldots, m$, both during dynamic transients and in steady state. It is easy to note that the singular value $\sigma_{i}$, for $i=1, \ldots, m$, is the weight parameter for the $i^{\text {th }}$ component of the tracking error in steady state. Since it is usually the case where $\sigma_{1}>\cdots \sigma_{k} \gg \sigma_{k+1}>\cdots>\sigma_{m}>0$, the input-output channels associated with the largest singular values are the most significant when minimizing $\bar{J}$ in (20). Therefore, it is possible that with the intent of minimizing $\bar{J}$ a lot of control effort will be spent to minimize the $i^{\text {th }}$ component of the tracking error, for $i>k$, which has a very small contribution to the overall value of the cost function. To avoid spending a lot of control effort for a marginal improvement of the cost function value, the singular value set is partitioned into significant singular values $S_{s}$ and negligible singular values $S_{n}$, i.e.,

$$
\begin{aligned}
& U=\left[\begin{array}{ll}
U_{s} & U_{n}
\end{array}\right], V=\left[\begin{array}{ll}
V_{s} & V_{n}
\end{array}\right], S=\left[\begin{array}{cc}
S_{s} & 0 \\
0 & S_{n}
\end{array}\right] \approx\left[\begin{array}{cc}
S_{s} & 0 \\
0 & 0
\end{array}\right], \\
& \Delta \bar{u}^{*}=\left[\begin{array}{l}
\Delta \bar{u}_{s}^{*} \\
\Delta \bar{u}_{n}^{*}
\end{array}\right], \Delta \bar{y}^{*}=\left[\begin{array}{l}
\Delta \bar{y}_{s}^{*} \\
\Delta \bar{y}_{n}^{*}
\end{array}\right] .
\end{aligned}
$$

The performance index defined in (20) can then be approximated as

$$
\bar{J} \approx \bar{J}_{s}=\sum_{i=1}^{k} \sigma_{i}^{2}\left(\Delta \bar{y}_{\text {tari }}^{*}-\Delta \bar{y}_{i}^{*}\right)^{2}=\left(\Delta \bar{y}_{\text {tar }}^{*}-\Delta \bar{y}_{s}^{*}\right)^{T} S_{s}^{2}\left(\Delta \bar{y}_{\text {tar }}^{*}-\Delta \bar{y}_{s}^{*}\right),
$$

where

$$
\begin{aligned}
\Delta \bar{y} & =Q^{-1 / 2} U S \Delta \bar{y}^{*} \approx Q^{-1 / 2} U_{s} S_{s} \Delta \bar{y}_{s}^{*} \Longleftrightarrow \Delta \bar{y}_{s}^{*}=S_{s}^{-1} U_{s}^{T} Q^{1 / 2} \Delta \bar{y} \\
\Delta \bar{y}_{\text {tar }} & =Q^{-1 / 2} U S \Delta \bar{y}_{\text {tar }}^{*} \approx Q^{-1 / 2} U_{s} S_{s} \Delta \bar{y}_{\text {tar }}^{*} \Longleftrightarrow \Delta \bar{y}_{\text {tar }}^{*}=S_{s}^{-1} U_{s}^{T} Q^{1 / 2} \Delta \bar{y}_{\text {tar }}, \\
\Delta \bar{u} & =R^{-1 / 2} V \Delta \bar{u}^{*}=R^{-1 / 2} V_{s} \Delta \bar{u}_{s}^{*}+R^{-1 / 2} V_{n} \Delta \bar{u}_{n}^{*} \Longleftrightarrow \Delta \bar{u}_{s}^{*}=V_{s}^{T} R^{1 / 2} \Delta \bar{u}, \\
\bar{e}_{s}^{*} & =\Delta \bar{y}_{\text {tars }}^{*}-\Delta \bar{y}_{s}^{*} .
\end{aligned}
$$

\subsection{Design of Robust MIMO Controller}

The SVD output and input bases reduce to $Q^{-1 / 2} U_{S} S_{S}$ and $R^{-1 / 2} V_{s}$, respectively. By defining

$$
\Delta y_{s}^{*}=S_{s}^{-1} U_{s}^{T} Q^{1 / 2} \Delta y, \quad \Delta u_{s}^{*}=V_{s}^{T} R^{1 / 2} \Delta u,
$$




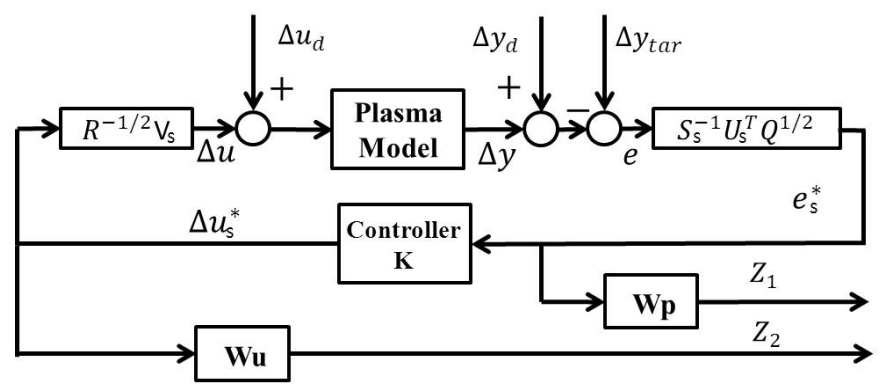

Figure 6: $H_{\infty}$ control formulation. Note that $W_{p}$ and $W_{u}$ are used only in the design of the controller $K$ to specify the performance criteria (they do not become part of the controller).

and using (8), we can write

$$
\frac{\Delta Y_{s}^{*}(s)}{\Delta U_{s}^{*}(s)}=P_{D C}=S_{s}^{-1} U_{s}^{T} Q^{1 / 2} P R^{-1 / 2} V_{s},
$$

where $\Delta Y_{s}^{*}(s)$ and $\Delta U_{s}^{*}(s)$ represent the Laplace transforms of the output $\Delta y_{s}^{*}$ and the input $\Delta u_{s}^{*}$, respectively. The transfer function $P_{D C}$ reduces in steady state (i.e., $s=0$ ) to a fully decoupled system in which there is a one-to-one relationship between the inputs $\Delta u_{s}^{*}$ and the outputs $\Delta y_{s}^{*}$. The plasma controller is synthesized based on this one-to-one relationship. The structure of the proposed controller is shown in Fig. 6, where two frequency-dependent functions, $W_{p}$ and $W_{u}$, are introduced to weight the tracking error and the control effort. The signals defined within the proposed controller structure are the control input $\Delta u_{s}^{*}$, the system output $\Delta y_{s}^{*}$, the tracking error $e_{s}^{*}=\Delta y_{\text {tar }}^{*}-\Delta y_{s}^{*}$, the target $\Delta y_{\text {tars }}^{*}=S_{s}^{-1} U_{s}^{T} Q^{1 / 2} \Delta y_{\text {tar }}$, the input disturbance $\Delta u_{d_{s}}^{*}=V_{s}^{T} R^{1 / 2} \Delta u_{d}$, the output disturbance $\Delta y_{d_{s}}^{*}=S_{s}^{-1} U_{s}^{T} Q^{1 / 2} \Delta y_{d}$, and the weighted performance signal $\left[Z_{1}^{T}, Z_{2}^{T}\right]^{T}=\left[\left(W_{p} e_{s}^{*}\right)^{T},\left(W_{u} \Delta u_{s}^{*}\right)^{T}\right]^{T}$. The transfer function $T_{z w}$ between $w=$ $\left[\Delta y_{\text {tars }}^{* T}, \Delta u_{d_{s}}^{* T}, \Delta y_{d_{s}}^{* T}\right]^{T}$ and $z=\left[Z_{1}^{T}, Z_{2}^{T}\right]^{T}$ for the closed-loop system shown in Fig. 6 can be expressed as

$$
\begin{aligned}
{\left[\begin{array}{l}
Z_{1} \\
Z_{2}
\end{array}\right]=\left[\begin{array}{c}
W_{p} e_{s}^{*} \\
W_{u} \Delta u_{s}^{*}
\end{array}\right] } & =\left[\begin{array}{ccc}
W_{p} M_{s} & -W_{p} M_{s} P_{D C} & -W_{p} M_{s} \\
W_{u} K M_{s} & -W_{u} K M_{s} P_{D C} & -W_{u} K M_{s}
\end{array}\right]\left[\begin{array}{c}
\Delta y_{\text {tar }}^{*} \\
\Delta u_{d_{s}}^{*} \\
\Delta y_{d_{s}}^{*}
\end{array}\right] \\
& =T_{z w}\left[\begin{array}{c}
\Delta y_{\text {tar }}^{*} \\
\Delta u_{d_{s}}^{*} \\
\Delta y_{d_{s}}^{*}
\end{array}\right]
\end{aligned}
$$


where $M_{S} \triangleq\left(I+P_{D C} K\right)^{-1}$ denotes the sensitivity transfer function. The transfer function $W_{p} M_{s}\left(-W_{p} M_{s}\right)$ represents the response of the weighted tracking error to the target (output disturbance), while the transfer function $-W_{p} M_{S} P_{D C}$ denotes the response of the weighted tracking error to the input disturbance. The transfer function $W_{u} K M_{s}\left(-W_{u} K M_{S}\right)$ represents the response of the weighted control effort to the target (output disturbance), while the transfer function $-W_{u} K M_{S} P_{D C}$ denotes the response of the weighted control effort to the input disturbance.

A controller $K(s)=\Delta U_{s}^{*}(s) / E_{s}^{*}(s)$, where $E_{s}^{*}(s)$ denotes the Laplace transform of $e_{s}^{*}$, is sought to stabilize the closed-loop system and minimize the $H_{\infty}$ norm of the transfer function $T_{z w}$, denoted as $\left\|T_{z w}\right\|_{\infty}$, i.e.,

$$
\min _{K(s)}\left\|T_{z w}\right\|_{\infty}
$$

The $H_{\infty}$ norm of the transfer function $T_{z w}$ is defined as the supremum over the frequency $\omega$ of the maximum singular value $\bar{\sigma}$ of the transfer function, i.e., $\left\|T_{z w}(s)\right\|_{\infty} \triangleq \sup _{\omega} \bar{\sigma}\left[T_{z w}(j \omega)\right]$. The $H_{\infty}$ norm represents the maximum energy amplification between input and output. Therefore, by minimizing $\left\|T_{z w}(s)\right\|_{\infty}$ while stabilizing the closed-loop system, the effect of the input signal (reference, input disturbance, output disturbance) on the energy of the output signal (frequencyweighted tracking error, frequency-weighted control effort) is also minimized. This is of critical importance because the objective is to keep the frequencyweighted tracking error $Z_{1}=W_{p} e_{s}^{*}$ and control effort $Z_{2}=W_{u} \Delta u_{s}^{*}$ small regardless of the characteristics of the reference $\Delta y_{\text {tars }}^{*}$, input disturbance $\Delta u_{d_{s}}^{*}$ and output disturbance $\Delta y_{d_{s}}^{*}$. Statement (26) defines what is called a mixed sensitivity $H_{\infty}$ control problem since the goal is twofold: reduction of the tracking error (minimization of $Z_{1}$ ) while using as little feedback control effort as possible (minimization of $Z_{2}$ ). The $H_{\infty}$ control synthesis technique is part of what is referred to as robust control theory [28] because by setting an upper bound for the $H_{\infty}$ norm of the closed-loop transfer function, the controller is able to guarantee stability and a level of performance regardless of changes in the plant dynamics within a predefined set. However, assessing the level of robustness both in terms of stability and performance would require first to quantify the model uncertainty, which is beyond the scope of this work. The goal of the control design procedure in this work is to achieve performance metrics in terms of error tracking and control effort minimization. Achieving robustness metrics, which would require incorporating an uncertainty model in the control design procedure, is not the control objective sought in this work. Therefore, any robustness property exhibited by the controller in experiments is not a direct consequence of the control design method. 


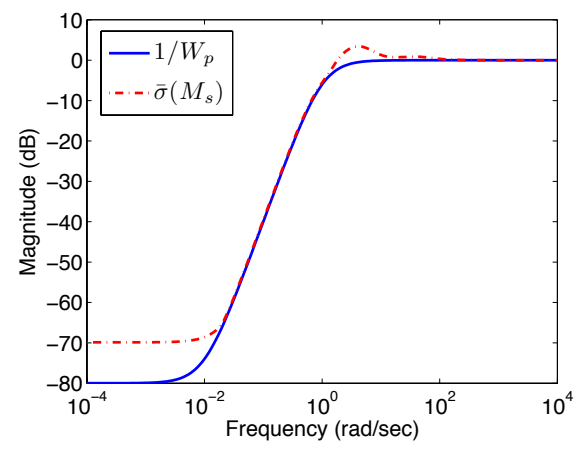

(a)

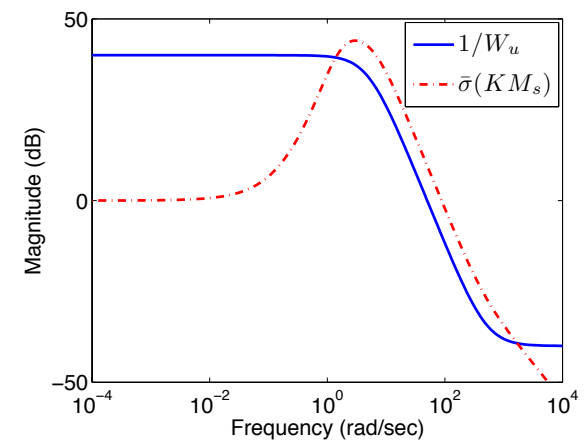

(c)

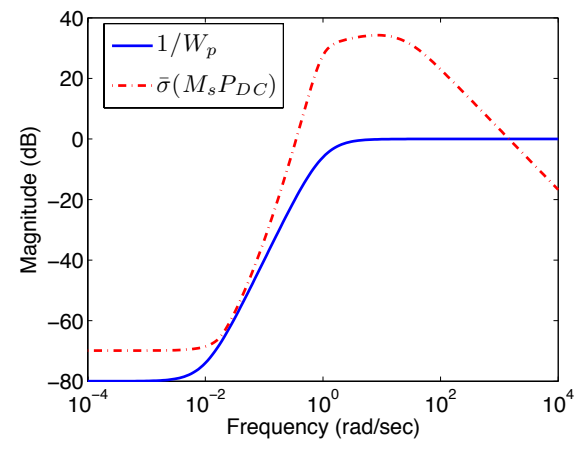

(b)

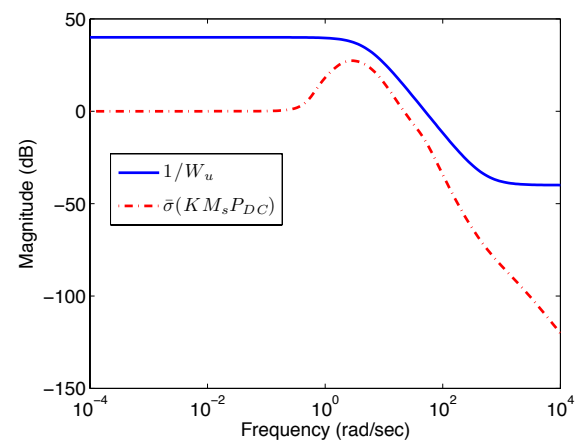

(d)

Figure 7: Inverse of performance weight $W_{p}$ and maximum singular values of transfer function $M_{s}$ (a) and $M_{S} P_{D C}$ (b). Inverse of performance weight $W_{u}$ and maximum singular values of transfer function $K M_{s}$ (c) and $K M_{s} P_{D C}$ (d).

The weighting functions $W_{p}$ and $W_{u}$, which can be parameterized as [27]

$$
W_{p}(s)=\left(\frac{\frac{s}{\sqrt{M_{1}}}+w_{b 1}}{s+w_{b 1} \sqrt{A_{1}}}\right)^{2} K_{p}, \quad W_{u}(s)=\left(\frac{s+w_{b 2} \sqrt{A_{2}}}{\frac{s}{\sqrt{M_{2}}}+w_{b 2}}\right)^{2} K_{u},
$$

are used to shape in the frequency domain the responses of the tracking error and control effort. The coefficients $M_{1}$ and $M_{2}$ are related to the high frequency behaviors, the parameters $A_{1}$ and $A_{2}$ are related to the low frequency behaviors, and $w_{b 1}$ and $w_{b 2}$ are related to the bandwidths of the transfer functions. The design parameters in the $H_{\infty}$ control synthesis, including $K_{p}$ and $K_{u}$, are shown in Table 1. The parameters are chosen to shape the frequency responses $1 /\left|W_{p}(j \omega)\right|$ and $1 /\left|W_{u}(j \omega)\right|$, which in turn serve as the desired upper bounds for the maximum singular values of the closed-loop transfer functions for the tracking error and control effort. For good reference tracking and low-frequency disturbance rejection, 
Table 1: The $H_{\infty}$ Control Parameters

\begin{tabular}{ccccc}
\hline Weight Function & $M_{i}$ & $w_{b i}$ & $A_{i}$ & $K_{i}$ \\
\hline$W_{p}$ & 1 & 1 & 0.0001 & 1 \\
$W_{u}$ & 100 & 10 & 0.01 & 1 \\
\hline
\end{tabular}

the magnitude of $M_{s}$ should approach zero at low frequency. In order to prevent amplification of high-frequency noise, the peak magnitude of $M_{s}$ needs to be suppressed and its magnitude should approach one at high frequency. Accordingly, for tight control the magnitude of $K M_{S}$ should be larger than one at low frequency, and should be attenuated below one at high frequency to avoid noise amplification by the feedback controller. Finally, the frequency range over which the feedback controller can actuate the system is specified by placing upper and lower limits on the bandwidth of the closed-loop system. Fig. 7 compares the frequency responses of the upper bounds $1 / W_{p}$ and $1 / W_{u}$ with the maximum singular values of the closed-loop transfer functions $M_{s}, M_{S} P_{D C}, K M_{s}$ and $K M_{s} P_{D C}$, achieved with the synthesized feedback controller $K$ (see transfer function (25)) for $R=$ $\operatorname{diag}([0.01,0.25,1000,0.5,0.25])$ and $Q=\operatorname{diag}([0.05,0.1,1,1,1,1])$. This figure shows to what extent the synthesized controller satisfies the desired specifications. As can be seen from Fig. 7, the frequency responses of the magnitudes of the maximum singular values of the closed-loop transfer functions stay in general below or slightly above their respective upper bounds within the frequency range of interest. In particular, Fig. 7 (a) shows that the achieved tracking capability of the controller is very close to the pursued tracking goal. From Fig. 7 (b), it is possible to note however that the controller cannot reject input disturbances in all frequencies. This is indeed expectable since it is not feasible to shape independently all the possible transfer functions in a closed loop system due to intrinsic sensitivity constraints $[27,28]$ and lack of degrees of freedom (note that that the transfer function between the target signal (or output disturbance) and the weighted tracking error in Fig. 7 (a) and the transfer function between the input disturbance and the weighted tracking error in Fig. 7 (b) share the same free parameter, which is the controller $K$ through the sensitivity function $M_{s}$, and are sought to be bounded by the same weight function $1 / W_{p}$ ). Importantly, the controller guarantees good tracking and effective rejection of input disturbances, which are constant in this work, at low frequencies, and no amplification of the output disturbance, which is usually noise, at high frequencies.

By solving the minimization problem (26), we can synthesize a controller $K$ that guarantees tracking while using as little control effort as possible. This is 
achieved both during the transients and in steady state, minimizing in turn (20). In practice, the control input and measured output of the original system $P$ are $\Delta u$ and $\Delta y$, respectively. The measured output is in turn used to compute the tracking error $e=\Delta y_{\text {tar }}-\Delta y$. As shown in Fig. 6, the overall $u$-profile and $\beta_{N}$ controller for system $P$ can be computed as

$$
\hat{K}(s)=\frac{\Delta U(s)}{E(s)}=R^{-1 / 2} V_{s} K(s) S_{s}^{-1} U_{s}^{T} Q^{1 / 2},
$$

where $\Delta U(s)$ and $E(s)$ denotes the Laplace transforms of $\Delta u$ and $e$, respectively, and where the definition of $K(s)$ and the relationships $e_{s}^{*}=S_{s}^{-1} U_{s}^{T} Q^{1 / 2} e, \Delta u=$ $R^{-1 / 2} V_{s} \Delta u_{s}^{*}$ have been used. For implementation in the DIII-D PCS, the controller (27) is expressed in state space form, i.e.,

$$
\begin{gathered}
\dot{x}_{c}=A_{c} x_{c}+B_{c}\left(\Delta y_{t a r}-\Delta y\right) \\
\Delta u=C_{c} x_{c}+D_{c}\left(\Delta y_{t a r}-\Delta y\right),
\end{gathered}
$$

where $x_{c}$ and $\left(A_{c}, B_{c}, C_{c}, D_{c}\right)$ denote the controller state vector and system matrices.

\subsection{Control Objectives, Expectations and Limitations}

The significant component of the tracking error $e_{s}^{*}$ introduced in the previous section and shown in Fig. 6 plays a crucial role both in understanding the objectives of the controller and in judging its performance. The significant component of the tracking error $e_{s}^{*}$, representing the difference between the significant components of the target $\Delta y_{\text {tar }}^{*}$ and the significant components of the output $\Delta y_{s}^{*}$, can be written as

$$
e_{s}^{*}=\Delta y_{\text {tar }}^{*}-\Delta y_{s}^{*}=S_{s}^{-1} U_{s}^{T} Q^{1 / 2}\left(\Delta y_{t a r}-\Delta y\right)=S_{s}^{-1} U_{s}^{T} Q^{1 / 2} e,
$$

where $e=\Delta y_{\text {tar }}-\Delta y=y_{\text {tar }}-y$ denotes the tracking error. It is critical to realize at this point that the dynamic component of the controller introduced in Section 3.3 is designed to reduce not the tracking error $e$ but the significant components of the tracking error $e_{s}^{*}$ as decided by the static component of the controller designed in Section 3.2, which takes care of the decoupling of the system and the selection of the significant control channels based on the significant basis given by the columns of $Q^{-1 / 2} U_{s} S_{s}$. The cost function

$$
J_{s}=\left(e_{s}^{*}\right)^{T} S_{s}^{2}\left(e_{s}^{*}\right)=\left(\Delta y_{\text {tar }}^{*}-\Delta y_{s}^{*}\right)^{T} S_{s}^{2}\left(\Delta y_{\text {tar }}^{*}-\Delta y_{s}^{*}\right)=\sum_{i=1}^{k} J_{s_{i}},
$$


which reduces to the cost function (22) as $t \rightarrow \infty$ and where $J_{s_{i}} \triangleq \sigma_{i}^{2}\left(\Delta y_{\text {tar }}^{*}\right.$ $\left.\Delta y_{i}^{*}\right)^{2}$, could therefore be used to evaluate the performance of the controller.

In other words, the objective of the controller is only to drive the component of the output $\Delta y$ lying in the subspace generated by the significant basis close to the component of the target $\Delta y_{\text {tar }}$ lying in the same subspace. The difference between these two components is indeed the part of the tracking error $e$ that can be driven to zero or removed by the controller if enough actuation is available. The tracking error can then be written as $e=e_{r m}+e_{n r m}$, where $e_{r m}$ is the removable tracking error and $e_{n r m}$ is the unremovable tracking error, which has no projection on the subspace generated by the significant basis, i.e. $S_{s}^{-1} U_{s}^{T} Q^{1 / 2} e_{n r m}=0$. The removable tracking error can be written as

$$
\begin{aligned}
e_{r m}=Q^{-1 / 2} U_{s} S_{s} e_{s}^{*}= & Q^{-1 / 2} U_{s} S_{s}\left[S_{s}^{-1} U_{s}^{T} Q^{1 / 2} e\right] \\
= & Q^{-1 / 2} U_{s} S_{s}\left[S_{s}^{-1} U_{s}^{T} Q^{1 / 2}\left(\Delta y_{\text {tar }}-\Delta y\right)\right] \\
= & Q^{-1 / 2} U_{s} U_{s}^{T} Q^{1 / 2} \Delta y_{\text {tar }}-Q^{-1 / 2} U_{s} U_{s}^{T} Q^{1 / 2} \Delta y \\
= & \left(Q^{-1 / 2} U_{s} U_{s}^{T} Q^{1 / 2} \Delta y_{\text {tar }}+y_{F F}\right) \\
& -\left(Q^{-1 / 2} U_{s} U_{s}^{T} Q^{1 / 2} \Delta y+y_{F F}\right) .
\end{aligned}
$$

The controller is then designed to reduce $e_{r m}$, or equivalently, to drive the significant output $\Delta y_{s}=Q^{-1 / 2} U_{s} U_{s}^{T} Q^{1 / 2} \Delta y$ close to the significant target $\Delta y_{\text {tars }}=$ $Q^{-1 / 2} U_{s} U_{s}^{T} Q^{1 / 2} \Delta y_{\text {tar }}$.

It is important to realize that, regardless of the number of significant singular vectors used for control design, the objective of the MIMO controller is not to achieve tight regulation for a finite number of components of the significant output but to reduce a removable tracking error that includes all the components of the output. The proposed controller is designed to drive the outputs as close as possible to their target values in order to minimize the overall weighted tracking error, which sometimes may result for instance in increasing the tracking error for some components of the output if this leads to a decrease of the tracking error for some other components that produces a net reduction of the overall weighted tracking error. It is therefore not expected individual and independent control of a finite number of points on the profile but a consistent reduction of the overall weighted tracking error. As the overall weighted tracking error is driven to zero, tight regulation of all the components of the output is achieved (in the subspace generated by the basis of significant singular vectors). However, it is unrealistic to expect no tracking error. As long as the control effort is limited by physical (actuation saturation) or design (actuation penalization) constraints, there will be 
some unreachable target output. Besides the saturation of the actuators, which is inherent to any physical system, in this work we penalize the control effort during both the static controller design (weighting matrix $R$ ) and the dynamic controller design (weighting function $W_{u}$ ). Therefore, neither the cost function (30) nor the removable error (31) will be driven to zero by the controller since its goal is not the pure elimination of tracking error but the combined minimization of tracking error and control effort.

It is also critical to understand that the controller is designed for a limited bandwidth. The weighting functions $W_{p}$ and $W_{u}$, which are functions of the frequency, not only impose relative weights on the tracking error and the control effort but also define the bandwidth over which the weights are imposed. In this way, the closed-loop transfer functions are shaped in the frequency domain with a particular bandwidth that will of course affect the response of the system. This can be appreciated from Fig. 7 (a), which shows the frequency response of the sensitivity function $M_{S}$ that relates the target signal with the tracking error. It is possible to note that for frequencies below $1 \mathrm{~Hz}$, the tracking error is attenuated, while for frequencies above $1 \mathrm{~Hz}$, it is not. This implies that the closed-loop system will be able to track only targets with frequency content below $1 \mathrm{~Hz}$. The bandwidth of the closed-loop system is directly correlated with the bandwidth of the open loop system, which is assumed during the modeling procedure by choosing the frequency content of the excitation (input) signals during the system identification experiments and the cutoff frequency of the low-pass filter.

Finally, it is always important to remember that the plasma response models used for control design in this work are linear and valid only around the reference state used during the system identification experiments. The linearity of the model limits the controller to applications where the control objective is just the regulation of the system around the reference state. Moreover, attempting just regulation around a reference state different from that used for system identification may limit the closed-loop performance.

\subsection{Design of Anti-windup Compensator}

The DIII-D tokamak is a nonlinear complex system, which is subject to actuator saturations as shown in Table 2. At the moment of designing the mixedsensitivity $H_{\infty}$ MIMO controller (28), the actuator saturations were not considered, i.e., $\hat{u}=u$ and $\Delta \hat{u}=\Delta u$. As a result of saturation, the actual plant input may be different from the output of the controller. In this case the controller output does not drive the plant input and, as a consequence, the states of the controller may 
Table 2: Actuator limits in DIII-D for current-profile control experiments (some limits are due to practical operating constraints while others (e.g., $I_{p}, P_{E C}$ ) are defined as administrative limits)

\begin{tabular}{ccccc}
\hline Channel & Actuator & Min & Max & Units \\
\hline 1 & Ip & 0.3 & 1.5 & MA \\
2 & Co-beam Power & 0 & 12.5 & MW \\
3 & Ct-beam Power & 0 & 5 & MW \\
4 & Blanced-beam Power & 0 & 2.5 & MW \\
5 & Total EC Power & 0.3 & 3 & MW \\
\hline
\end{tabular}

wind up because the plant does not respond as expected, which can cause the behavior of the system to deteriorate dramatically or even become unstable. To cope with the negative effects of saturation, the goal is not to redesign the proposed MIMO controller but to design an anti-windup compensator that keeps the controller well-behaved and avoid undesirable oscillations when saturation is present. The anti-windup compensator must in addition leave the nominal closed-loop unmodified when no saturation is present. The anti-windup augmentation is written as

$$
\begin{aligned}
\dot{x}_{a w} & =A_{a w} x_{a w}+B_{a w}(\hat{u}-u)+\gamma(u, \hat{u}) \lambda \\
s & =C_{a w} x_{a w} \\
\lambda & =-c x_{a w}-A_{a w} x_{a w}-B_{a w}(\hat{u}-u)
\end{aligned},
$$

where $\hat{u}$ denotes the output of the saturation function defined as

$$
\hat{u}_{i}=\operatorname{sat}_{u_{i}^{\text {max }}}^{u_{\text {max }}}\left(u_{i}\right)=\left\{\begin{array}{ll}
u_{i}^{\text {max }} & \text { if } u_{i}^{\text {max }}<u_{i} \\
u_{i} & \text { if } u_{i}^{\text {min }}<u_{i}<u_{i}^{\text {max }}, \\
u_{i}^{\text {min }} & \text { if } u_{i}<u_{i}^{\text {min }}
\end{array},\right.
$$

where $u_{i}^{\max }$ and $u_{i}^{\min }$ are the maximum and minimum saturation limits for $i^{\text {th }}$ input channel, for $i=1, \ldots, m, \gamma(u, \hat{u})=1$ if $u=\hat{u}$ and 0 otherwise, and $c$ is a positive constant. The system matrices $\left(A_{a w}, B_{a w}, C_{a w}\right)$ of the anti-windup compensator are chosen identical to the system matrices $(A, B, C)$ of the plant model $P$ in (5)(6) $[29,30]$.

\subsection{Control Algorithm Implementation in the DIII-D PCS}

The controller was implemented as a discrete-time state-space system with a sampling time of 20 milliseconds. The motional Stark effect (MSE) beam used to obtain $q$ profile measurements in real-time was turned on for 10 milliseconds and then off for 10 milliseconds within each sampling period. The rtEFIT algorithm uses the MSE measurements to provide $\beta_{N}$, the plasma current $I_{p}$, the poloidal stream function at the magnetic axis $\psi_{a x i s}$ and at the plasma boundary $\psi_{b d r y}$, and 
Table 3: Initial Conditions

\begin{tabular}{ccccc}
\hline Shot & Identification & Reference & Dist. I & Dist. II \\
\hline$I_{p}$ & $0.9045 \mathrm{MA}$ & $0.9013 \mathrm{MA}$ & $0.8957 \mathrm{MA}$ & $0.8972 \mathrm{MA}$ \\
$\beta_{N}$ & 1.8840 & 2.3897 & 2.4097 & 2.3763 \\
\hline
\end{tabular}

the safety factor $q$ on a normalized flux spatial domain $\psi_{n}=\frac{\psi-\psi_{\text {axis }}}{\psi_{b d r y}-\psi_{\text {axis }}}$. By using the relationship between the toroidal flux and the mean effective minor radius, a coordinate transformation algorithm (see Appendix D in [20]) has been implemented in the PCS as part of this work to construct the to-be-controlled magnetic profile $(q, \imath, \Psi=2 \pi \psi$, or $\theta \triangleq \partial \psi / \partial \hat{\rho})$ as a function of the normalized mean effective minor radius $\hat{\rho}$ from the data provided by the rtEFIT algorithm.

\section{Closed-loop Simulated and Experimental Results}

Simulated and experimental results are presented in this section to illustrate the performance of the proposed feedforward + feedback control scheme. The whole control system, which combines the MIMO controller and the anti-windup compensator, is shown in Fig. 3. A reference (feedforward) control shot \#146417 for the control experiment was run first without feedback control in order to reproduce flat-top conditions of the system identification experiments. Fig. 8 compares $l$ and $q$ at $t=2.5 \mathrm{~s}$ for the typical system identification shot \#140090 and the reference control shot \#146417, and Table 3 provides the values of $I_{p}$ and $\beta_{N}$ at $t=2.5 \mathrm{~s}$ for both shots. Both the figure and the table show that the reproduction is not completely successful, neither in terms of the $q$ or $l$ profiles, nor in terms of $\beta_{N}$, which can impact the closed-loop performance due to the questionable validity of the linear model. The feedforward inputs (after $t=2.5 \mathrm{~s}$ ), denoted as $u_{F F}$, for the reference control shot \#146417, which are represented by red dashed lines in the figures in this section, are $I_{p}=0.9 \mathrm{MA}, P_{C O}=1.9838 \mathrm{MW}, P_{C T}=0$ $\mathrm{MW}, P_{B L}=2 \mathrm{MW}$, and $P_{E C}=1.4415 \mathrm{MW}$. The feedforward inputs are rather different from those used during the system identification experiments, which are given by $I_{p}=0.85 \mathrm{MA}, P_{C O}=3.2681 \mathrm{MW}, P_{C T}=0 \mathrm{MW}, P_{B L}=0 \mathrm{MW}$, and $P_{E C}=1.4431 \mathrm{MW}$ (after $t=2.5 \mathrm{~s}$ ). This choice was driven by the decision of: (i) moving the feedforward input values away from the saturation values and providing more headroom for the feedback controller while approximately preserving the total NBI power; (ii) creating a to-be-tracked target profile slightly different from the system-identification reference profile (assuming good reproduction of system-identification conditions at $t=2.5 \mathrm{~s}$ ). The $\imath$ profile and $\beta_{N}$ resulting from these reference (feedforward) inputs in shot $\# 146417$, denoted as $y_{F F}$, are used 


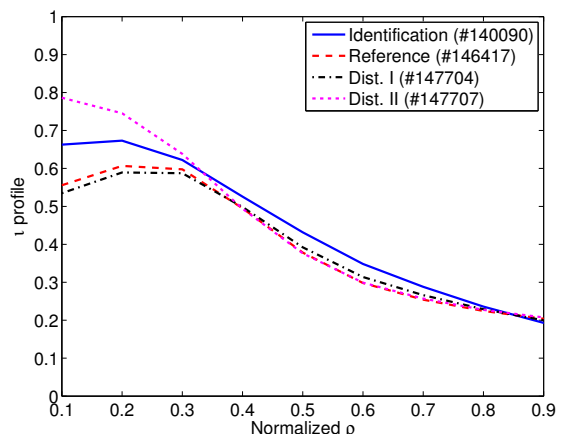

(a)

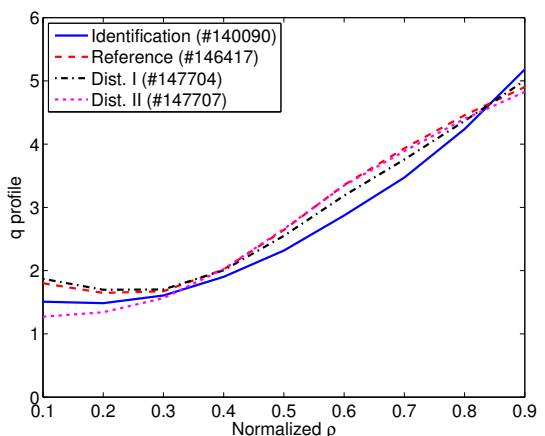

(b)

Figure 8: Rotational transform $\imath$ profile (a) and safety factor $q$ profile (b) for shots \#140090, $\# 146417, \# 147704$ and \#147707 at $t=2.5 \mathrm{~s}$.

Table 4: Input Disturbances

\begin{tabular}{cccccc}
\hline Number & $\Delta I_{p_{d}}$ & $\Delta P_{C O_{d}}$ & $\Delta P_{C T_{d}}$ & $\Delta P_{B L_{d}}$ & $\Delta P_{E C_{d}}$ \\
\hline Disturbance I & $0.1 \mathrm{MA}$ & $0 \mathrm{MW}$ & $0 \mathrm{MW}$ & $0 \mathrm{MW}$ & $0 \mathrm{MW}$ \\
Disturbance II & $0.1 \mathrm{MA}$ & $0.1 \mathrm{MW}$ & $0 \mathrm{MW}$ & $-0.1 \mathrm{MW}$ & $0 \mathrm{MW}$ \\
\hline
\end{tabular}

as targets in the control experiments and represented by red dashed lines in the figures in this section.

The goal for both simulations and experiments was to demonstrate that the proposed controller is capable of regulating the system around target $\boldsymbol{l}$ profile and $\beta_{N}$ even in the presence of disturbances. Note that since the control goal is regulation, $\Delta y_{\text {tar }} \equiv 0 \Longleftrightarrow y_{\text {tar }}=y_{F F}$ in all the control experiments. As discussed in Section 3.4, this implies that $\Delta y_{t a r_{s}}=\Delta y_{\text {tar }} \equiv 0$ and $e_{r m}=y_{F F}-\left(\Delta y_{s}+y_{F F}\right)$. Since the goal of the controller is to drive $e_{r m}$ close to zero while minimizing the control effort, it is therefore of interest to compare $\Delta y_{s}+y_{F F}$ with $y_{\text {tar }}\left(=y_{F F}\right)$, which are represented by black dashed-dotted lines and red dashed lines, respectively, in the figures in this section. Two sets of input disturbances (shown in Table 4) were created for this purpose. The input disturbances of the plasma current and $\mathrm{H} \& \mathrm{CD}$ powers can be expressed as $\Delta u_{d}=\left[\Delta I_{p_{d}}^{T}, \Delta P_{C O_{d}}^{T}, \Delta P_{C T_{d}}^{T}, \Delta P_{B L_{d}}^{T}, \Delta P_{E C_{d}}^{T}\right]^{T}$ (see Fig. 3 or Fig. 6 for disturbance injection point). Disturbance I, representing a disturbance in the plasma current, is introduced at $t=3 \mathrm{~s}$ to test $t$ profile control. Disturbance II, representing disturbances in both the plasma current and the neutral beam powers, is introduced at $t=3 \mathrm{~s}$ to test simultaneous $\imath$ profile and $\beta_{N}$ control. Note that while the total neutral beam power will be kept constant by Disturbance II, it is expected that the disturbance in the plasma current will in turn introduce a disturbance in $\beta_{N}$. Moreover, the disturbance in the plasma 
current will affect the outer region of the $\imath$ profile while the disturbances in the beam powers will affect the inner region of the $\imath$ profile.

The decision of not smoothening the target signal $y_{t a r}\left(=y_{F F}\right)$ obtained as a direct measurement from the reference (feedforward) control shot \#146417, although not optimal and leading to a more complicated analysis of the results, responds to the convenience of minimizing operations between discharges to avoid implementation mistakes and to maximize the experimental time. The objective is however by no means the tracking of the measurement noise. The high-frequency noise cannot be indeed tracked by the closed-loop system due to its limited bandwidth (see Fig. 7 (a)). It is therefore expected that most of the noisy component of the target be filtered by the closed-loop system itself and approximately the same closed-loop response be obtained regardless of using a noisy or a smoothened target signal.

During the control experiments, the counter-injection beam was not available and the gyrotrons were either poorly controlled or unavailable. The unavailability of the counter-injection beam was known right before the experiment, allowing for the redesign of the controllers in preparation for this condition by setting a high value for the associated weight in the $R$ matrix. Associating a high weight to an unavailable actuator instead of removing it from the model, which would indeed be a better solution, allows for the redesign of the controller without modifying its number of outputs, reducing in this way the risk of controller implementation problems when the actuator is lost right before the experiment. The gyrotrons were lost during the experiment, not allowing for the redesign of the controller. Note that the controller does not request counter-injection beam power but it does request gyrotron power.

The proposed controllers were tested in the flat-top phase of the discharge, from $t_{i}=2.5 \mathrm{~s}$ to $t_{f}=6 \mathrm{~s}$ in both simulations and experiments. To allow for comparison between simulations and experiments, the same reference (feedforward) inputs, input disturbances, actuator constraints, and approximate initial plasma conditions were used in both cases.

\subsection{Case 1: Rotational Transform i Profile Control under Disturbance I}

When the objective is the control of just the $\imath$ profile, only the state equation of the slow model (5) is used for control design. To improve the tracking performance while avoiding spending a lot of control effort for a marginal improvement of the cost function value, the control weight matrix is defined as $R=\operatorname{diag}([0.01,0.25,1000,0.5,0.25])$ and only the two most significant singular values $(k=2)$ are preserved in this case study. In this way, the plasma current 

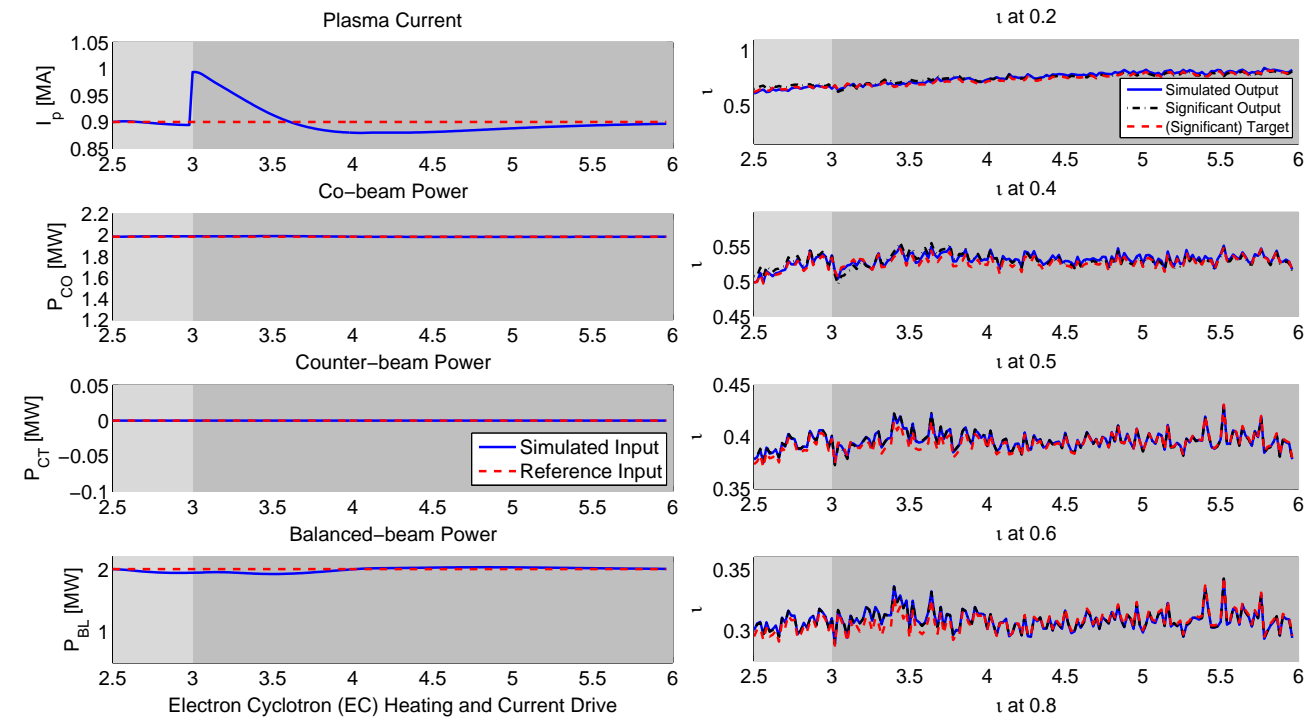

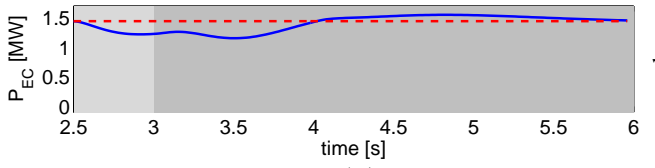

(a)

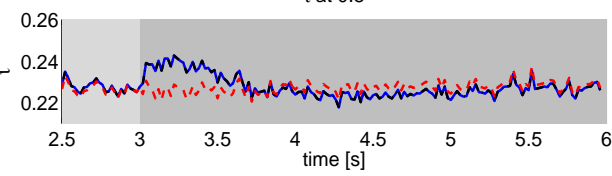

(b)

Figure 9: Rotational transform $\imath$ profile closed-loop-controlled simulation (shot \#147704): (a) Inputs, (b) Outputs. Disturbance I. Light-gray background: feedback on - disturbance off, darkgray background: feedback on - disturbance on.

$I_{p}$ plays a significant role in controlling the $\imath$ profile at the plasma boundary and the effort by the controller is focused on reducing the two most significant contributions to the cost function value. Note that this decision is rather conservative because up to $k=3$ singular values could have been kept according to the number of available actuators $\left(I_{p}, P_{C O}, P_{B L}\right)$. Equal weight is put on each tracking error component, i.e., $Q=\operatorname{diag}([1,1,1,1,1])$.

Fig. 9 shows simulation results. The controller rejects the disturbance in the plasma current $I_{p}$ quickly and drives the $I_{p}$ close to the constant reference (feedforward) value, as shown in Fig. 9 (a.1). Beam and gyrotron powers, shown in Fig. 9 (a.2)-(a.5), are weakly modulated by the feedback controller within the saturation limits and are driven towards to their reference (feedforward) values by the end of the discharge. In the first 0.5 second of the simulation, from $t=2.5 \mathrm{~s}$ to $t=3 \mathrm{~s}$, the regulation results for the undisturbed plant are satisfactory for all control points $(\hat{\rho}=0.2,0.4,0.5,0.6,0.8)$ as shown in Fig. 9 (b). Once the distur- 


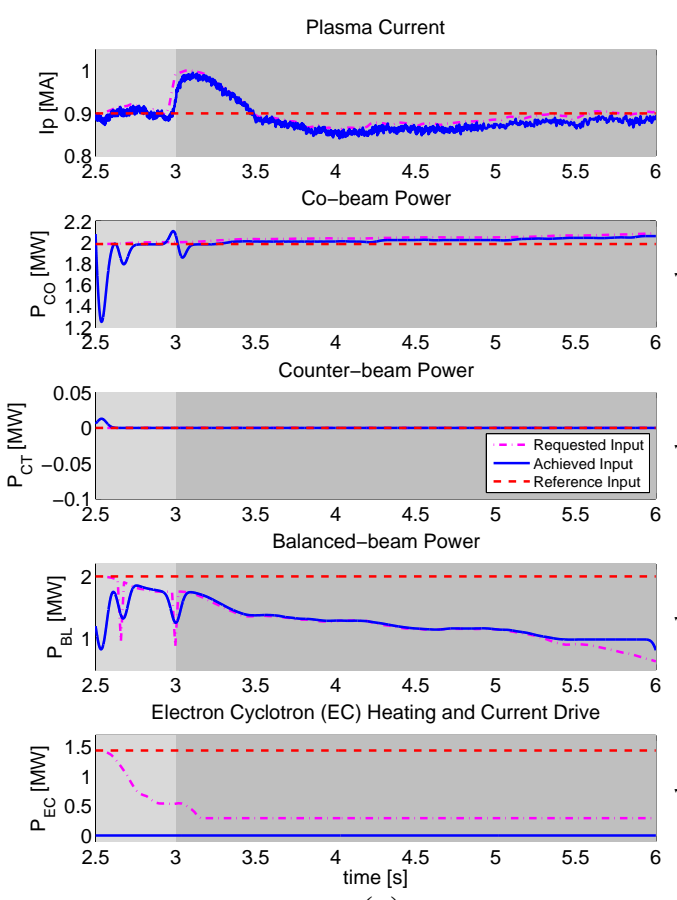

(a)
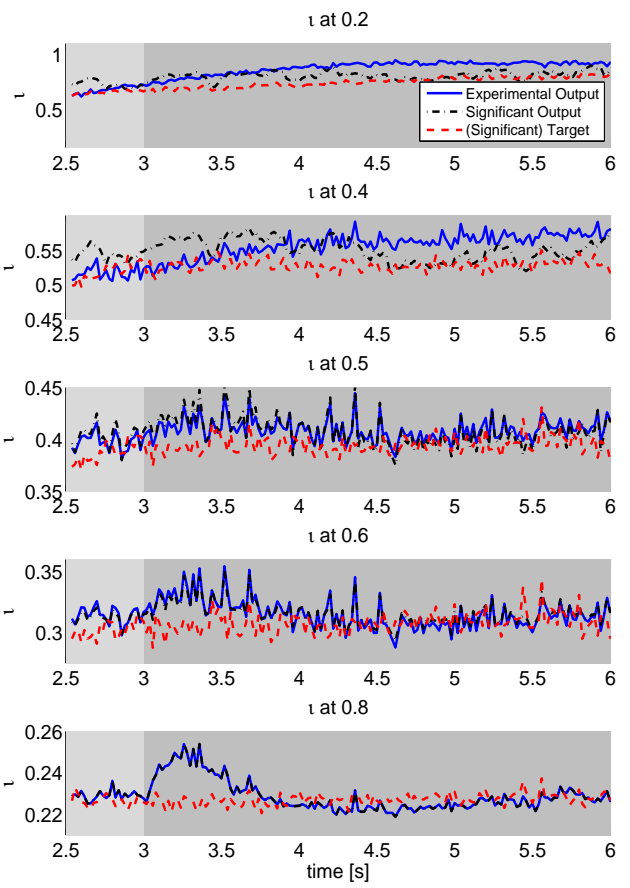

(b)

Figure 10: Rotational transform $\imath$ profile closed-loop-controlled experiment (shot \#147704): (a) Inputs, (b) Outputs. Disturbance I. Light-gray background: feedback on - disturbance off, darkgray background: feedback on - disturbance on.

bance in the plasma current is injected at $t=3 \mathrm{~s}$, its effect on the outer $\imath$ profile becomes clearly notable from Fig. 9 (b.5), where it is shown that the $\imath$ value at $\hat{\rho}=0.8$ ramps up. The robust controller rejects the disturbances and reduces the tracking errors in less than one second, keeping them below 5\% for the rest of the discharge.

Fig. 10 shows the experimental results obtained from shot \#147704. As shown in Fig. 10 (a.1), the $t$-profile controller rejects the plasma current disturbance rather fast and the dedicated $I_{p}$ controller delivers the requested current very closely. The working beams successfully follow the values requested by the 1 profile controller but the EC H\&CD system is not available during the discharge and does not deliver the requested value, which introduces an additional disturbance into the closed-loop system that the controller must overcome. Both the EC H\&CD system and the counter-injection beam have important effects on the plasma resistivity and toroidal current density in the center of the plasma. This can be appreciated by examining the steady-state gains of the system in Fig. 4, where 


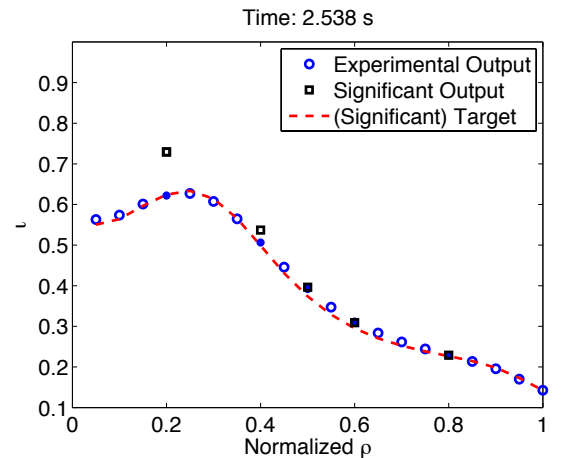

(a) Feedback ON, Disturbance OFF

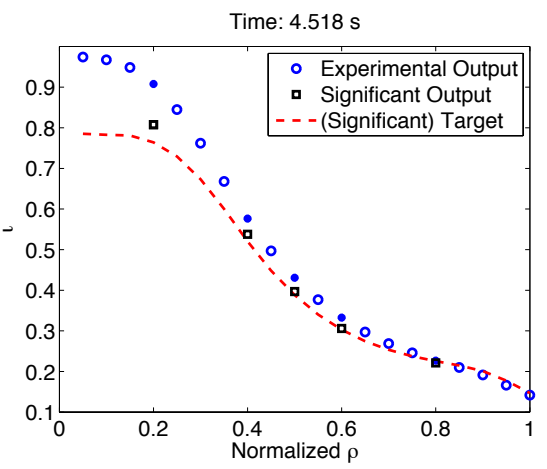

(c) Feedback ON, Disturbance ON

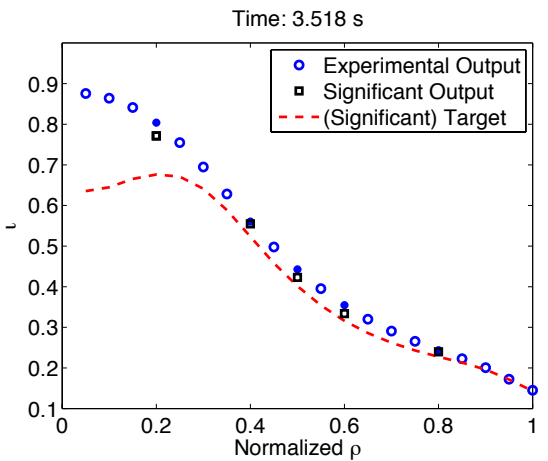

(b) Feedback ON, Disturbance ON

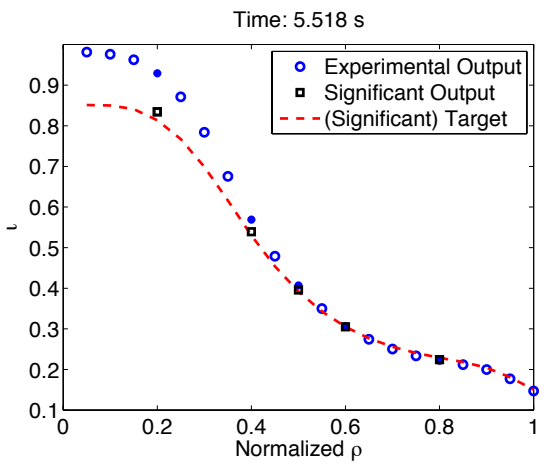

(d) Feedback ON, Disturbance ON

Figure 11: Plasma $t(\hat{\rho})$ profile at time $t=2.538,3.518,4.518,5.518 \mathrm{~s}$ (shot \# 147704). Target (red dashed line), significant $l$ (black squares) and experimental $l$ (blue circles) profiles.

both $P_{C T}$ and $P_{E C}$ strongly affect the inner part of the steady-state $\imath$ profile. The lack of EC H\&CD power regulation, added to the unavailability of the counterinjection beam, makes the control of the inner $l$ profile very challenging. Since the plasma current disturbance is quickly rejected after $t=3 \mathrm{~s}$, the outer profile tracking errors are kept small. There are nearly no tracking errors after $t=4 \mathrm{~s}$ for $l$ at $\hat{\rho}=0.5,0.6,0.8$ as noted from Fig. 10 (b.3)-(b.5). During the experiment, the requested EC and balanced-beam powers reach saturation and activate the antiwindup compensator, which causes the difference observed between simulation (Fig. 9) and experiment (Fig. 10).

A series of four plasma $\imath$ profiles at different times during the experiment are shown in Fig. 11. As can be seen from Fig. 11 (a), the initial profile is very close to the target, which implies that the conditions of shot \# 146417 have been successfully reproduced at $t=2.5 \mathrm{~s}$. After the input disturbances is injected into 

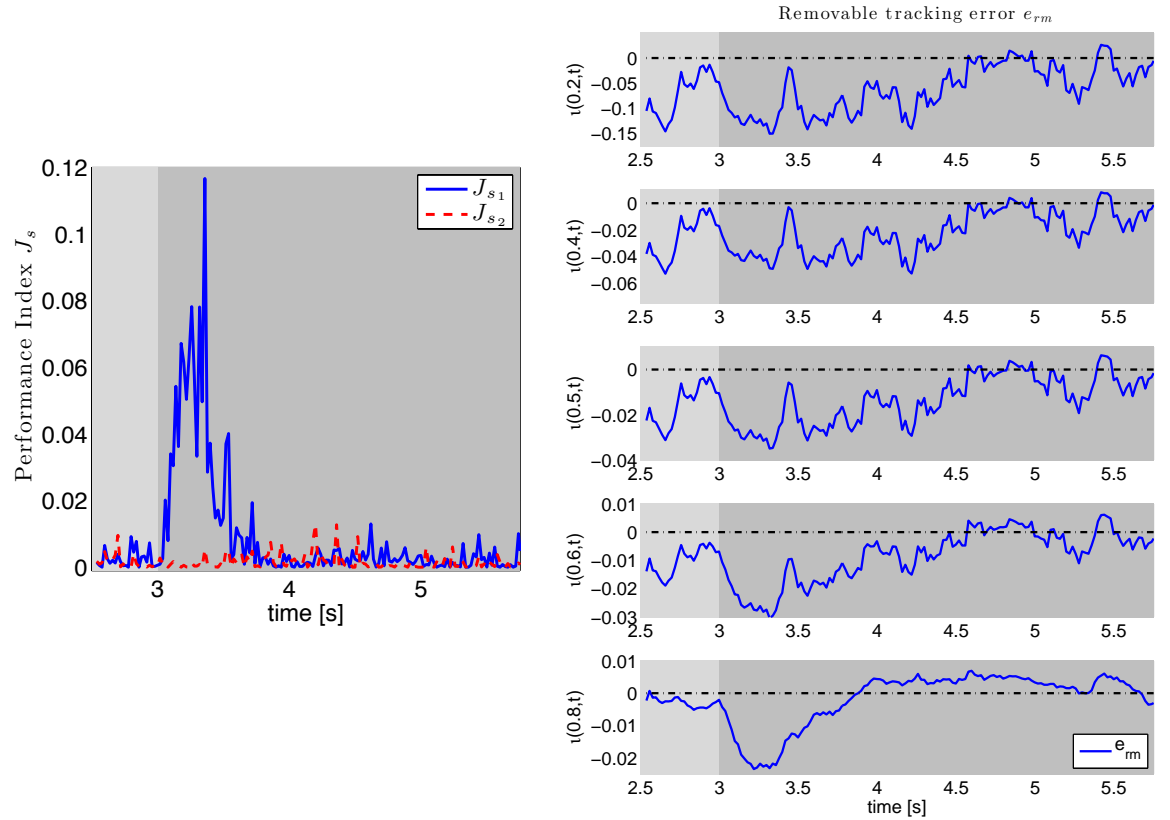

(a)

(b)

Figure 12: Control performance metrics (shot \#147704 - Disturbance I): (a) Cost function, (b) Removable tracking error for $\imath$ profile at $\hat{\rho}=0.2,0.4,0.5,0.6,0.8$. Light-gray background: feedback on - disturbance off, dark-gray background: feedback on - disturbance on.

the system, the tracking performance deteriorates, particularly in the inner region of the plasma as shown in Fig. 11 (b). As time goes on, the controller rejects the disturbance and forces the tracking errors at $\hat{\rho}=0.5,0.6,0.8$ to become smaller as shown in Fig. 11 (c)-(d). Due to the unavailability of both the EC H\&CD and the counter-injection NBI systems in this experiment, the tracking performance at $\hat{\rho}=0.2,0.4$ remains rather poor during the whole discharge.

Since $(k=2)<(m=p=5)$ in this case, not all the outputs live in the subspace generated by the significant singular vectors. In other words, $\Delta y_{s} \neq \Delta y$ and not all the tracking error is removable $\left(e=e_{r m}+e_{n r m}\right)$. This explains the fact that blue solid lines and dashed-dotted black lines are not coincident in Fig. 10, and blue circles and black squares are not coincident in Fig. 11. It is however possible to note from these figures that the two dominant output singular vectors can indeed reproduce quite well the outer part of the profile, i.e., $\Delta y_{s} \approx \Delta y$ in the outer region. This is not the case in the in inner region, where there is a marked difference between $\Delta y_{s}$ and $\Delta y$, and therefore a substantial unremovable tracking error. This can be explained by examining the steady-state gains of the system 
in Fig. 4, where the responses of the system to both $P_{C O}$ and $P_{B L}$, the only beam groups available in this experiment, show a marked difference between $\Delta \bar{y}_{s}$ and $\Delta \bar{y}$ in the inner region and a good match in the outer region. Therefore, it is not only the unavailability of $P_{E C}$ and $P_{C T}$ but also the inability of $P_{C O}$ and $P_{B L}$ to make $\Delta y_{s} \approx \Delta y$ in the inner region the reasons for a poor tracking performance at $\hat{\rho}=0.2,0.4$ as shown in Fig. 10 and Fig. 11. It is important to note at this point that by shaping the singular vector bases, the weighting matrices $Q$ and $R$ play an important role in determining the differences between $\Delta \bar{y}_{s}$ and $\Delta \bar{y}$ for the different actuators observed in Fig. 4. However, what is important from the point of view of the controller performance is its ability to drive $\Delta y_{s}$ close to zero, or $\Delta y_{S}+y_{F F}$ (dashed-dotted black lines) close to $y_{t a r}=y_{F F}$ (red dashed lines), as shown in Fig. 10 for all the points of the $l$ profile, including those in the inner region $(\hat{\rho}=0.2$ and $\hat{\rho}=0.4)$. This can also be appreciated from Fig. 11, where the significant output profile $\Delta y_{s}+y_{F F}$ (black squares) is driven close to the target profile $y_{t a r}=y_{F F}$ (red dashed lines). The time evolution of the significant components of the cost function $J_{S}$ (30) in Fig. 12 (a) shows the effectiveness of the controller in rejecting the disturbance within the subspace generated by the dominant singular vectors. Note that the effect of the disturbance is mainly captured by the most dominant output singular vector. This can be explained by noting that Disturbance I (see Table 4) heavily affect the most dominant input singular vector (see Fig. 5 (b)). Fig. 12 (b) shows that all the removable tracking error $e_{r m}$ (31) components are driven close to zero.

\subsection{Case 2: Rotational Transform 1 Profile and $\beta_{N}$ Control under Disturbance II}

Due to the large difference in plasma resistivity between the center and the edge, the current density rapidly equilibrates at the edge, and evolves slowly in the center. This, combined with the actuation limitations discussed in Section 4.1, makes the control of the inner $l$ profile very challenging. Recognizing the lack of capability for controlling the inner part of the profile, and to prevent the controller from spending a large amount of control effort through the available actuators trying to reduce the tracking error in the inner region without any significant improvement in overall performance, the state weight matrix is chosen as $Q=\operatorname{diag}([0.05,0.1,1,1,1,1])$ (the weights of the tracking errors associated with $\imath(0.2, t)$ and $t(0.4, t)$ are reduced). The main control effort is therefore applied to $\imath(0.5, t), \imath(0.6, t), \imath(0.8, t)$, and $\beta_{N}(t)$. Moreover, in order to be able to evaluate the controller effectiveness in regulating the $\imath$ profile and $\beta_{N}(t)$ within the limited controllable region defined by the available actuators, only the two most important singular values $(k=2)$ are preserved in this case study. Note that 

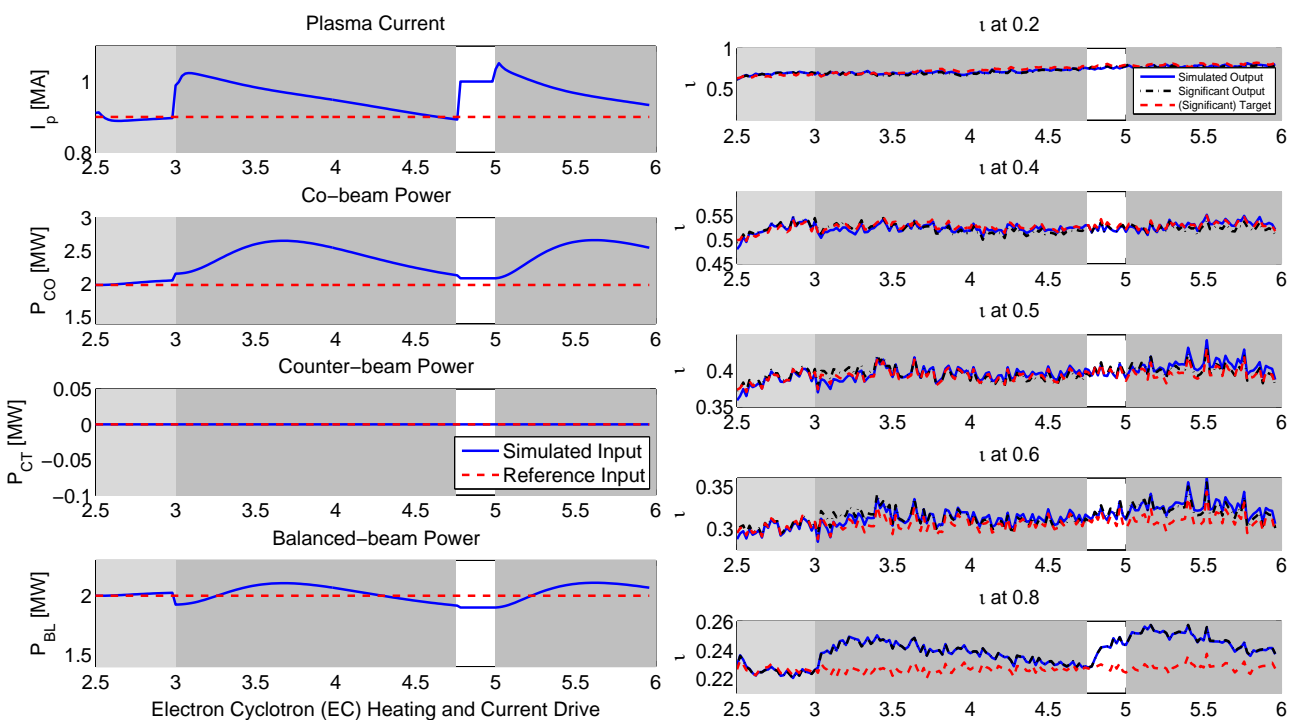

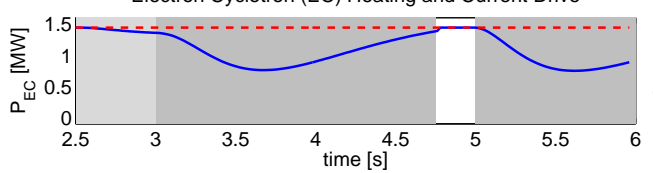

(a)
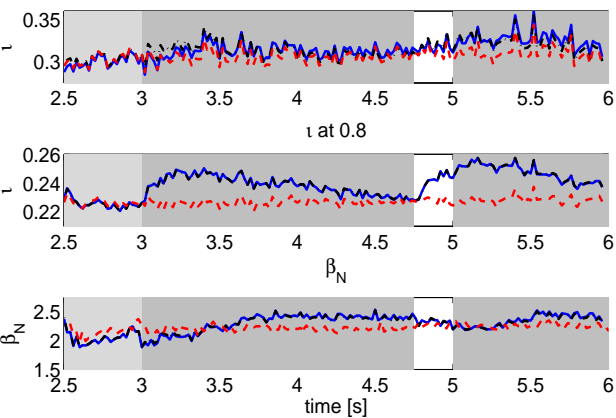

(b)

Figure 13: Rotational transform $\imath$ profile closed-loop-controlled simulation (shot \#147707): (a) Inputs, (b) Outputs. Disturbance II. Light-gray background: feedback on - disturbance off, dark-gray background: feedback on - disturbance on, white background: feedback off - disturbance on.

this decision, as in the previous case, is rather conservative because up to $k=3$ singular values could have been preserved. The expectation is that the three available actuators will provide the capability of actuating in these two most dominant directions. The other control parameters are the same as in Section 4.1. The feedback controller is turned on and off throughout the discharge, i.e., OFF for 0 to $2.5 \mathrm{~s}$, ON for 2.5 to $4.75 \mathrm{~s}$, OFF for 4.75 to $5 \mathrm{~s}$, and ON for 5 to $6 \mathrm{~s}$.

Fig. 13 shows simulation results. In the first 0.5 second of the simulation, from $t=2.5 \mathrm{~s}$ to $t=3 \mathrm{~s}$, the controller regulates $\imath$ and $\beta_{N}$ around the target values. When the disturbance is initially introduced into the system at $t=3 \mathrm{~s}$, the outer $t$ profile moves away from the target value immediately. As shown in Fig. 13 (a.1), the disturbance in the plasma current is rejected by the controller and the reference (feedforward) value is recovered after the transient. Beam and gyrotron powers, shown in Fig. 13 (a.2)-(a.5), are modulated by the feedback controller away from their reference values without hitting saturation limits. Due to the increased level 
of beam power, $\beta_{N}$ also moves away from its target value. The feedback controller finally rejects the effects of the input disturbance after around $1.75 \mathrm{~s}$, and both the target $l$ profile and $\beta_{N}$ evolutions are once again effectively tracked. Then the controller is turned off for 0.25 second from $t=4.75 \mathrm{~s}$ to $t=5 \mathrm{~s}$, and the tracking error becomes larger in the outer region of the $l$ profile (there is no large change in the neutral beam powers after turning off the feedback controller and the disturbance in the plasma current seems to modify $\beta_{N}$ in the expected direction). In the final second of the simulation, from $t=5 \mathrm{~s}$ to $t=6 \mathrm{~s}$, the controller is turned back on and it rejects nearly all the effects of the disturbance by repeating the control actions already observed during the first on-period from $t=3 \mathrm{~s}$ to $t=4.75 \mathrm{~s}$. Based on the results from Fig. 13, we can note that the outer $t$ profile is strongly affected by the plasma current and $\beta_{N}$ is strongly affected by the beam and gyrotron powers. The controller tries to recover the target $l$ profile without producing large $\beta_{N}$ excursions. Once the disturbance is rejected, the $\imath$ profile and $\beta_{N}$ are driven to the target values.

Fig. 14 shows the experimental results obtained from shot \#147707. The plasma current (Fig. 14 (a.1)) and the beams (Fig. 14 (a.2)-(a.4)) successfully follow the requested values without exhibiting any saturation. The EC (Fig. 14 (a.5)), used for plasma heating and current drive, is off during the experiment. The difference between achieved and requested values of $P_{E C}$ (Fig. 14 (a.5)) can be interpreted as an additional disturbance because, as explained before, the controller has not been redesigned to avoid using the EC H\&CD system and still requests a $P_{E C}$ value. It can be noted from Fig. 15 (a) that it is indeed not possible to reproduce the target profile in the center of the plasma ( $l$ at $\hat{\rho}=0.2)$ at the beginning of the closed-loop control experiment $(t=2.5 \mathrm{~s})$, i.e., the conditions of the reference control shot \#146417 at $t=2.5 \mathrm{~s}$ have not been successfully reproduced in this case. This is also reflected in the initial condition of the time trace for $l$ at $\hat{\rho}=0.2$ in Fig. 14 (b). It may be important to appreciate from Fig. 8 that the initial profile is closer in this case to that achieved in the system identification shot \#140090 at $t=2.5$. From $t=3 \mathrm{~s}$ to $t=4.75 \mathrm{~s}$, the controller rejects the input disturbance very effectively. Note from Fig. 14 (b) how both the $\imath$ profile and $\beta_{N}$ recover their target values after the transient produced by the injection of disturbances at $t=3 \mathrm{~s}$. This is in part due to the fact that the achieved control inputs follow the requested values very well for the working actuators. When the controller is turned off at $t=4.75 \mathrm{~s}$, the actuator values drift away from the feedforward values immediately and a tracking error becomes noticeable particularly for $\beta_{N}$ and the outer $\imath$ profile. Finally, the feedback controller is turned on at $t=5 \mathrm{~s}$ and it drives back the $\imath$ profile and $\beta_{N}$ to their target values, rejecting once again the effects of the 

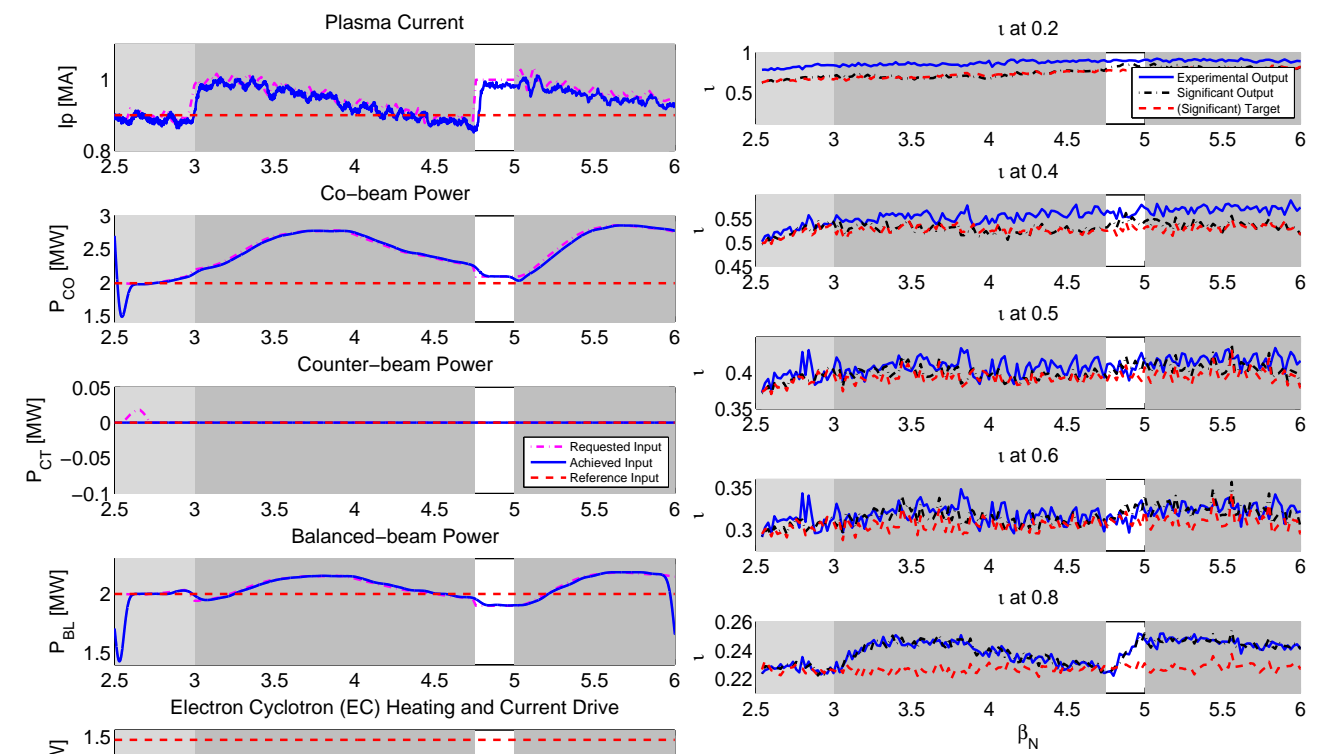

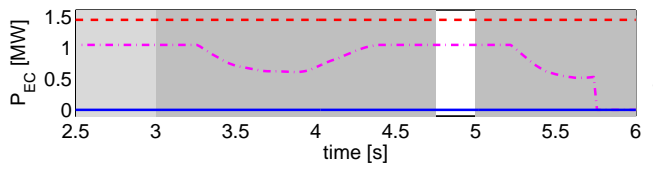

(a)

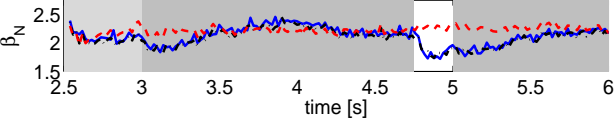

(b)

Figure 14: Rotational transform $\imath$ profile closed-loop-controlled experiment (shot \#147707): (a) Inputs, (b) Outputs. Disturbance II. Light-gray background: feedback on - disturbance off, dark-gray background: feedback on - disturbance on, white background: feedback off - disturbance on.

input disturbance. When the experimental input and output signals in Fig. 14 are compared with those in Fig. 13, which are predicted by the identified model in closed-loop simulations, it is possible to observe a remarkable similarity. Since the input signals (Fig. 13 (a) and Fig. 14 (a)) are determined exclusively by the controller based on the measurements provided by the diagnostics, the observed similarity is another proof of the capability of the identified model to capture the plasma dynamics around the regulated state.

A series of six plasma profiles at different times of the shot \#147707 are shown in Fig. 15. Due to the design of the weight matrix $Q$, the control effort is mainly applied to $\imath(0.5, t), \imath(0.6, t), \imath(0.8, t)$ and $\beta_{N}$. The $\imath$ tracking errors in the center of the plasma are larger than the $l$ tracking errors at the edge of plasma. This is in part explained by the fact that, as shown in Fig. 15 (a), the target profile is not reproduced at the initial time probably due to the loss of critical actuators (counter-injection NBI and EC powers). After the input disturbances are injected 


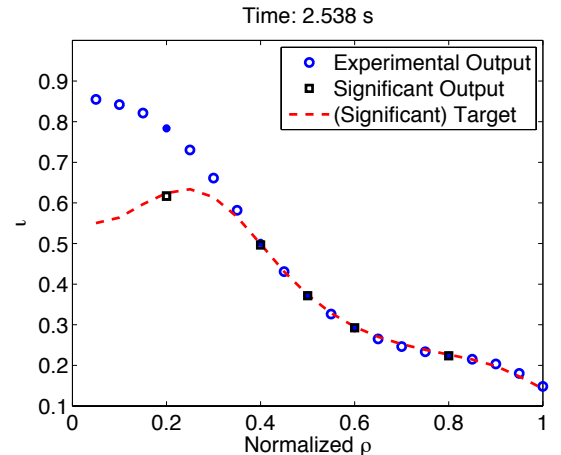

(a) Feedback ON, Disturbance OFF

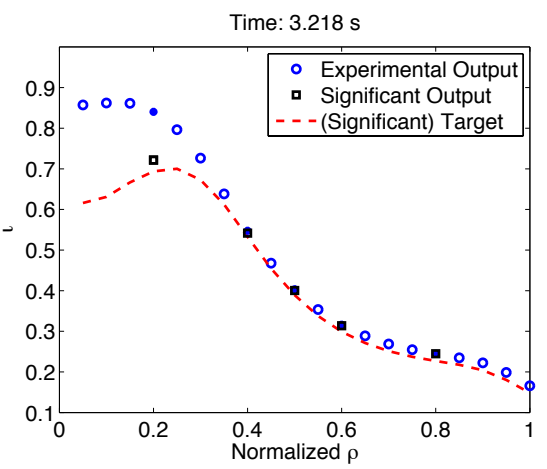

(c) Feedback ON, Disturbance ON

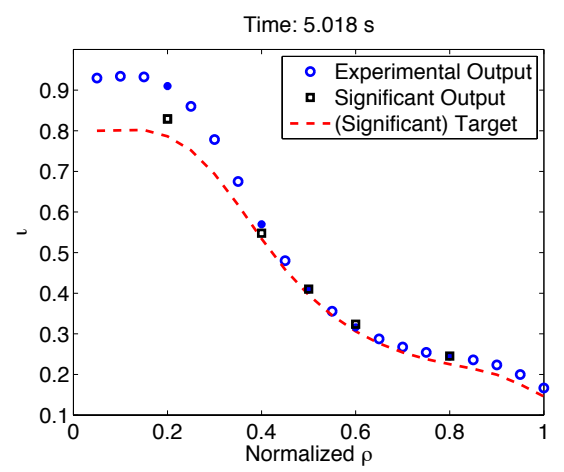

(e) Feedback ON, Disturbance ON

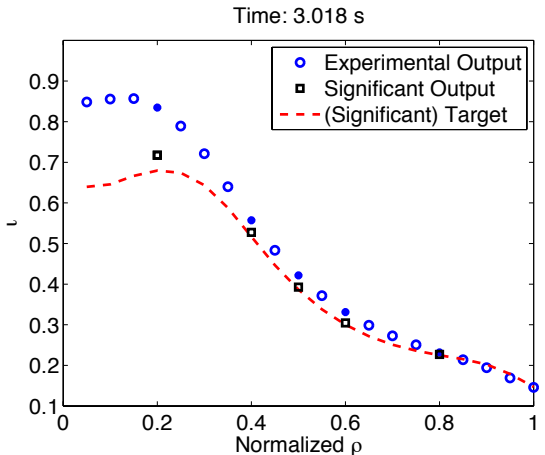

(b) Feedback ON, Disturbance OFF

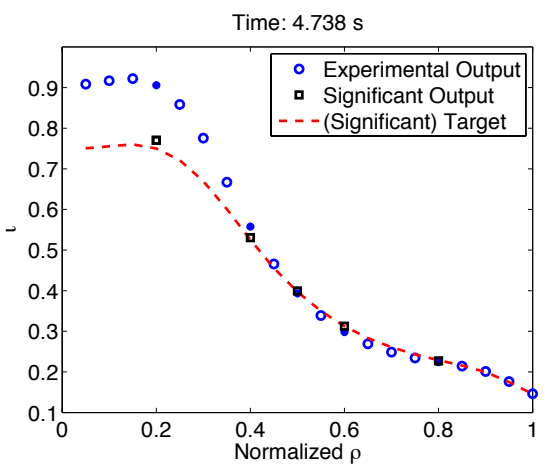

(d) Feedback ON, Disturbance ON

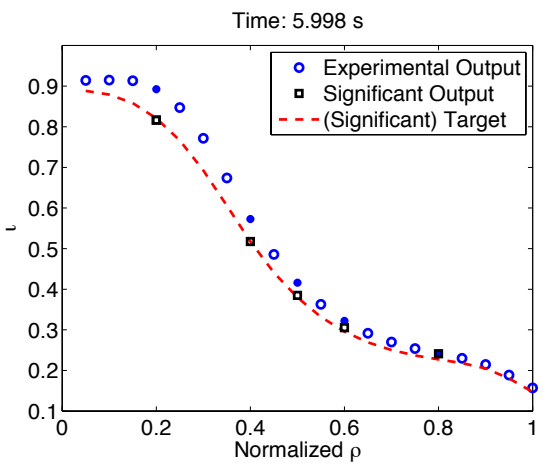

(f) Feedback ON, Disturbance ON

Figure 15: Plasma $t(\hat{\rho})$ profile at time $t=2.538,3.018,3.218,4.738,5.018,5.998 \mathrm{~s}$ (shot \# 147707). Target (red dashed line), significant $\iota$ (black squares) and experimental $\iota$ (blue circles) profiles. 

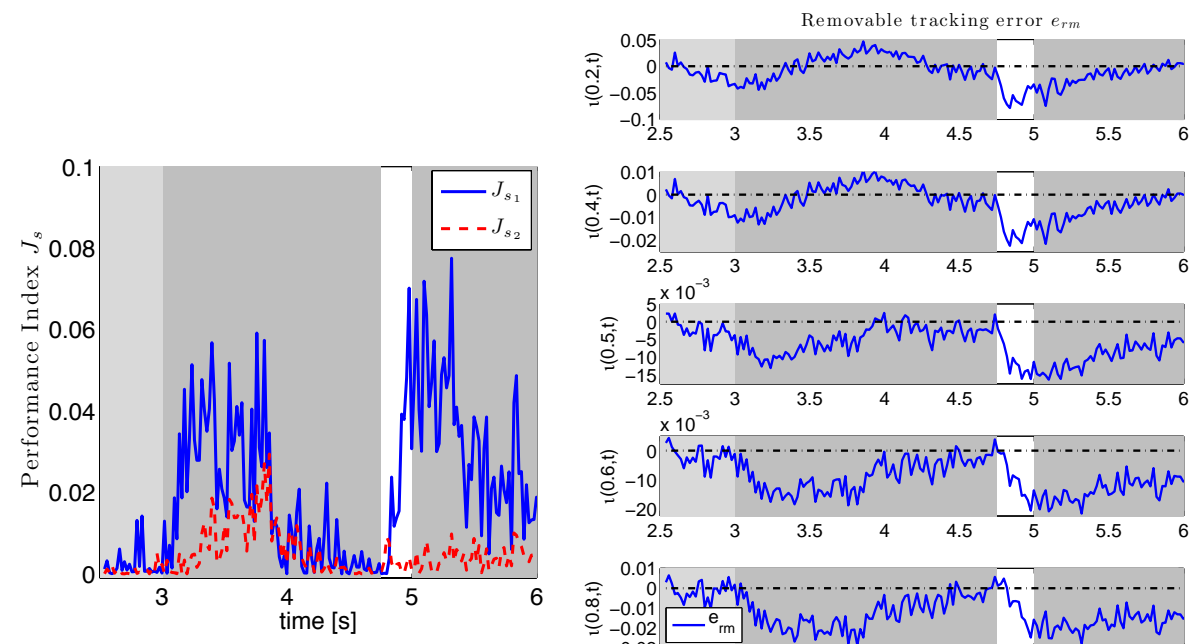

2.5

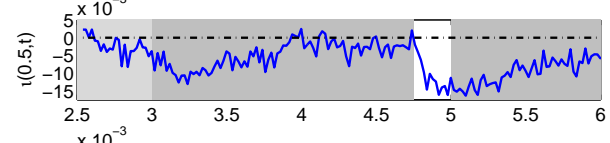

$\times 10^{-3}$
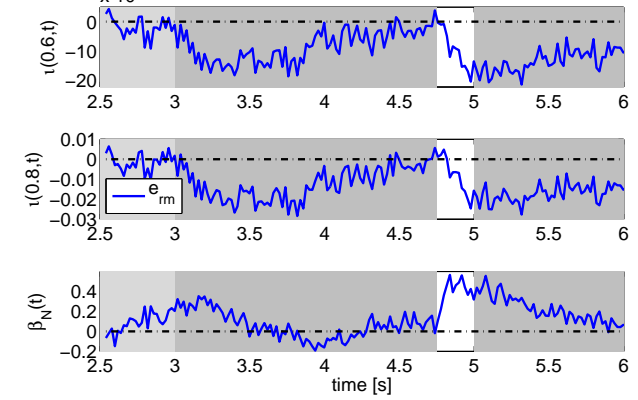

(a)

(b)

Figure 16: Control performance metrics (shot \#147707 - Disturbance II): (a) Cost function, (b) Removable tracking error for $\imath$ profile at $\hat{\rho}=0.2,0.4,0.5,0.6,0.8$ and $\beta_{N}$. Light-gray background: feedback on - disturbance off, dark-gray background: feedback on - disturbance on, white background: feedback off - disturbance on.

into the system, these tracking errors become larger, as shown in Fig. 15 (b). As time goes on in shot $\# 147707$, the tracking errors become smaller as shown in Fig. 15 (c), (d) thanks to the action of the feedback controller. When the feedback controller is turned off, the tracking errors increase once again as shown in Fig. 15 (e) before recovering after the controller is turned back on as shown in Fig. 15 (f).

Since $(k=2)<(m=5),(p=6)$ in this case, not all the outputs live in the subspace generated by the significant singular vectors. Therefore, as in the previous case, $\Delta y_{s} \neq \Delta y$ and not all the tracking error is removable $\left(e=e_{r m}+e_{n r m}\right)$. This explains the fact that blue solid lines and dashed-dotted black lines are not coincident in Fig. 14, and blue circles and black squares are not coincident in Fig. 15. Similarly to the previous case, the two dominant output singular vectors can indeed reproduce quite well the outer part of the profile, i.e., $\Delta y_{s} \approx \Delta y$ in the outer region. This is not the case in the in inner region, where there is a marked difference between $\Delta y_{s}$ and $\Delta y$, and therefore a substantial unremovable tracking error. What is important from the point of view of the controller perfor- 
mance is its ability to drive $\Delta y_{s}$ close to zero, or $\Delta y_{s}+y_{F F}$ (dashed-dotted black lines) close to $y_{\text {tar }}=y_{F F}$ (red dashed lines), as shown in Fig. 14 for all the points of the $\imath$ profile. This can also be appreciated from Fig. 15, where the significant output profile $\Delta y_{s}+y_{F F}$ (black squares) is driven close to the target profile $y_{\text {tar }}=y_{F F}$ (red dashed lines). Note, as a difference from the previous case, that the removable component of the error is relatively small during all the discharge in the inner region $(\hat{\rho}=0.2$ and $\hat{\rho}=0.4)$, i.e., $\Delta y_{s}+y_{F F} \approx y_{\text {tar }}=y_{F F}$ for $\hat{\rho}=0.2$ and $\hat{\rho}=0.4$. This may be related to the selection made for the components of the matrix $Q$ associated with these outputs, which makes almost the entirety of the error unremovable freeing the controller from the responsibility of tightly regulating the $\imath$ profile at these points. The time evolution of the significant components of the cost function $J_{s}(30)$ in Fig. 16 (a) shows the effectiveness of the controller in rejecting the disturbance within the subspace generated by the dominant singular vectors. Note that in this case the effect of the disturbance is captured by the two most dominant output singular vectors since Disturbance II (see Table 4) projects not only on the first but also on the second most dominant input singular vector (see Fig. 5 (b)). Fig. 16 (b) shows that all the removable tracking error $e_{r m}$ (31) components are driven close to zero.

\section{Conclusions}

A robust, model-based, MIMO, $\imath$-profile and $\beta_{N}$ controller has been designed for the flat-top phase of DIII-D H-mode discharges. The design is based on a two-timescale linear, dynamic, plasma-response model, which has been identified around a reference profile during the current flat-top phase. The feedback controller is designed based on this model to regulate the system around a target, which is assumed to be close to the reference profile around which the model has been identified, even in the presence of various disturbances. Singular value decomposition of the steady state transfer function is used to decouple the system and identify the most relevant control channels. The mixed sensitivity $H_{\infty}$ technique is used to minimize the tracking error and to optimize control effort ignoring the saturation. Then the closed-loop system is augmented with an anti-windup compensator in order to minimize the effects of any control input constraint. The proposed controller represents one of the first profile controllers integrating magnetic and kinetic variables ever implemented and experimentally tested in DIII-D.

The preliminary experimental results presented in this work, although limited in number and constrained by actuators problems, show good progress towards routine current profile control in DIII-D and leaves valuable lessons for control 
redesign. The controller has been proven effective at reducing the removable component of the tracking error, which has been defined as the part of the tracking error that can be driven to zero based on the control authority given by the number of significant singular values retained during the selection of the most relevant control channels. The number of significant singular values should be no greater than the number of available actuators. Demonstration of full profile control is still pending and will require the availability of all the actuators and the use of all the control channels.

Some rather sluggish and weak actuation observed in the closed-loop experiment may not be related to the inability of the controller to actuate in some directions because of limited actuation or neglected control channels but to the limited bandwidth of the closed-loop system. More aggressive controllers could be designed in this case by increasing the frequency content of the excitation (input) signals during the system identification experiments, by increasing the cutoff frequency, or even better, by eliminating the need to choose a cutoff frequency associated with the slow dynamics of the system during the identification of the datadriven model, by increasing the weight $Q$ and decreasing the weight $R$ (tighter control at the expense of more control effort) during the design of the static component of the controller, and by selecting weights $W_{p}$ and $W_{u}$ leading to an increase of the closed-loop response bandwidth during the design of the dynamic component of the controller. The anti-windup compensator would be an indispensable companion of these more aggressive controllers. In addition, it would be critical to simultaneously control $\beta_{N}$ while controlling the $\imath$ profile to prevent the controller from triggering MHD instabilities in its aggressive effort to achieve the desired target profile. The risk of triggering MHD instabilities in our search for a faster response is however not reserved to an aggressive feedback control action. As we introduce more vigorous excitations in order to increase the bandwidth of the data-driven response model, we also increase the likelihood of triggering MHD instabilities during the open-loop system identification experiments, which clearly represents another challenge associated with the design of data-driven controllers.

More experimental tests are needed to assess the appropriateness of using datadriven linear models for current profile control. Being able to control the current profile during the ramp-up and ramp-down phases, being able to regulate the current profile for different scenarios (around different reference states), or being able to drive the current profile from one target profile to another will most likely require adaptive or nonlinear control approaches based on richer dynamic models obtained by a first-principles-driven modeling approach. However, the preliminary control experiments carried out at DIII-D suggest that regulation of the cur- 
rent profile around a reference state during the flat-top phase of the discharge, as is the objective of this work, may be possible using a data-driven linear modeling and control approach if enough actuation is available. However, a serious study on the performance of the regulator as the target state moves away from the reference state around which the linear model has been identified is still pending. In relation to the control approach proposed in this work, the sensitivity of the static component of the controller, which decides the most relevant control channels by a SVD approach, to un-modeled or mis-modeled plasma response and its impact on performance need further analysis.

\section{Acknowledgment}

This work was supported by the National Science Foundation CAREER Award program (ECCS-0645086), the U.S. Department of Energy (DE-FG02-09ER55064 and DE-FC02- 04ER54698) and the European Communities under the contract of Association between EURATOM and CEA (this work was carried out within the framework of the European Fusion Development Agreement. The views and opinions expressed herein do not necessarily reflect those of the European Commission).

\section{References}

[1] J. Lister, A. Portone, Y. Gribov, Plasma Control in ITER, IEEE Control Systems Magazine 26 (2) (2006) 79-91.

[2] T. Wijnands, D. Houtte, G. Martin, X. Litaudon, P. Froissard, Feedback control of the current profile on Tore Supra, Nuclear Fusion 37 (6) (1997) 777.

[3] O. Barana, D. Mazon, L. Laborde, F. Turco, Feedback control of the lower hybrid power deposition profile on Tore Supra, Plasma Physics and Controlled Fusion 49 (7) (2007) 947.

URL http://stacks.iop.org/0741-3335/49/i=7/a=001

[4] T. Suzuki, A. Isayama, S. Ide, T. Fujita, T. Oikawa, S. Sakata, M. Sueoka, H. Hosoyama, M. Seki, J. Team, Recent RF experiments and application of RF waves to real-time control of safety factor profile in JT-60U, AIP Conference Proceedings 787 (1) (2005) 279-286. doi:10.1063/1.2098241. URL http: / / link.aip.org/link/?APC/787/279/1 
[5] T. Suzuki, S. Ide, T. Oikawa, T. Fujita, M. Ishikawa, M. Seki, G. Matsunaga, T. Hatae, O. Naito, K. Hamamatsu, M. Sueoka, H. Hosoyama, M. Nakazatoand, J. Team, Off- axis current drive and real-time control of current profile in JT-60U, Nuclear Fusion 48 (4) (2008) 045002.

[6] J. Ferron, et al., Feedback Control of the Safety Factor Profile Evolution during Formation of an Advanced Tokamak Discharge, Nuclear Fusion 46 (10) (2006) L13-17.

[7] Y. Ou, T. Luce, E. Schuster, et al., Towards Model-based Current Profile Control at DIII-D, Fusion Engineering and Design 82 (2007) 1153-1160.

[8] E. Witrant, et al., A control-oriented model of the current profile in tokamak plasma, Plasma Physics and Controlled Fusion 49 (2007) 1075-1105.

[9] F. Felici, et al., Real-time physics-model-based simulation of the current density profile in tokamak plasmas, Nuclear Fusion 51 (083052).

[10] Y. Ou, C. Xu, E. Schuster, et al., Design and simulation of extremum-seeking open-loop optimal control of current profile in the DIII-D tokamak, Plasma Physics and Controlled Fusion 50 (115001).

[11] C. Xu, J. Dalessio, Y. Ou, E. Schuster, et al., Ramp-Up Phase Current Profile Control of Tokamak Plasmas via Nonlinear Programming, IEEE Transactions on Plasma Science 38 (2) (2010) 163-173.

[12] F. Felici, O. Sauter, Non-linear model-based optimization of actuator trajectories for tokamak plasma profile control, Plasma Physics and Controlled Fusion 54 (025002).

[13] Y. Ou, C. Xu, E. Schuster, Robust Control Design for the Poloidal Magnetic Flux Profile Evolution in the Presence of Model Uncertainties, IEEE Transactions on Plasma Science 38 (3) (2010) 375-382.

[14] Y. Ou, C. Xu, E. Schuster, et al., Optimal Tracking Control of Current Profile in Tokamaks, IEEE Transactions on Control Systems Technology 19 (2) (2011) 432-441.

[15] C. Xu, Y. Ou, E. Schuster, Sequential linear quadratic control of bilinear parabolic PDEs based on POD model reduction, Automatica 47 (2) (2011) $418-426$. 
[16] Y. Ou, C. Xu, E. Schuster, T. Luce, J. Ferron, M. Walker, D. Humphreys, Receding-horizon optimal control of the current profile evolution during the ramp-up phase of a tokamak discharge, Control Engineering Practice 19 (2011) 22-31.

[17] S. H. Kim, J. B. Lister, A potentially robust plasma profile control approach for ITER using real-time estimation of linearized profile response models, Nuclear Fusion 52 (2012) 074002.

[18] F. Bribiesca Argomedo, E. Witrant, C. Prieur, S. Brémond, R. Nouailletas, J. Artaud, Lyapunov-based distributed control of the safety factor profile in a tokamak plasma, Nuclear Fusion 53 (2013) 033005.

[19] O. Gaye, E. Moulay, S. Bremond, L. Autrique, R. Nouailletas, J. Artaud, Y. Orlov, Robust stabilization of the current profile in tokamak plasmas using sliding mode approach in infinite dimension, Control Engineering Practice 21 (2013) 1350-1358.

[20] J. Barton, M. Boyer, W. Shi, E. Schuster, et al., Toroidal current profile control during low confinement mode plasma discharges in DIII-D via firstprinciples-driven model-based robust control synthesis, Nuclear Fusion 52 (2012) 123018.

[21] M. Boyer, J. Barton, E. Schuster, T. Luce, J. Ferron, M. Walker, D. Humphreys, B. Penaflor, R. Johnson, First-principles-driven model-based current profile control for the DIII-D tokamak via LQI optimal control, Plasma Physics and Controlled Fusion 55 (2013) 105007.

[22] M. Boyer, J. Barton, E. Schuster, M. Walker, T. Luce, J. Ferron, B. Penaflor, R. Johnson, D. Humphreys, Backstepping control of the toroidal plasma current profile in the DIII-D tokamak, IEEE Transactions on Control Systems Technology 22 (5) (2014) 1725-1739.

[23] L. Ljung, System Identification: Theory for the User, Prentice Hall, New Jersey, 1999.

[24] D. Moreau, et al., Real-time Control of the q-profile in JET for Steady State Advanced Tokamak Operation, Nuclear Fusion 43 (2003) 870-882. 
[25] D. Moreau, et al., A Two Time Scale Dynamic Model Approach for Magnetic and Kinetic Profile Control in Advanced Tokamak Scenarios on JET, Nuclear Fusion 48 (106001).

[26] D. Moreau, et al., Plasma Models for Real-time Control of Advanced Tokamak Scenarios, Nuclear Fusion 51 (063009).

[27] S. Skogested, I. Postlethwaite, Multivariable Feedback Control, John Wiley and Sons, Ltd, New York, 2003.

[28] K. Zhou, J. C. Doyle, Essentials of Robust Control, Prentice Hall, New Jersey, 1997.

[29] L. Zaccarian, A. R. Teel, Modern Anti-windup Synthesis: Control Augmentation for Actuator Saturation, Princeton University Press, New Jersey, 2004.

[30] E. Schuster, M. Walker, D. Humphreys, M. Kristic, Plasma Vertical Stabilization with Actuation Constraints in the DIII-D Tokamak, Automatica 41 (2005) 1173-1179.

[31] W. Shi, W. Wehner, J. Barton, M. D. Boyer, E. Schuster, et al., Multivariable Robust Control of the Plasma Rotational Transform Profile for Advanced Tokamak Scenarios in DIII-D, in: American Control Conference (ACC), 2012, 2012, pp. 5037-5042.

[32] W. Wehner, W. Shi, E. Schuster, et al., Optimal Feedback Control of the Poloidal Magnetic Flux Profile in the DIII-D Tokamak based on Identified Plasma Response Models, in: Proceeding of the 2012 American Control Conference, 2012, pp. 5049-5054.

[33] W. Shi, W. Wehner, J. Barton, M. Boyer, E. Schuster, et al., A Two-timescale Model-based Combined Magnetic and Kinetic Control System for Advanced Tokamak Scenarios on DIII-D, in: Proceeding of the 51st IEEE International Conference on Decision and Control, 2012, pp. 4347-4352.

[34] D. Moreau, M. Walker, J. Ferron, F. Liu, E. Schuster, J. Barton, M. Boyer, K. Burrell, S. Flanagan, P. Gohil, R. Groebner, C. Holcomb, D. Humphreys, A. Hyatt, R. Johnson, R. L. Haye, J. Lohr, T. Luce, J. Park, B. Penaflor, W. Shi, F. Turco, W. Wehner, the ITPA-IOS group members and experts, Integrated magnetic and kinetic control of advanced tokamak plasmas on DIII-D based on data-driven models, Nuclear Fusion 53 (2013) 063020. 
[35] C. A. J. Fletcher, Computational Galerkin Methods, Springer-Verlag, New York, 1984.

[36] W. Wehner, C. Xu, E. Schuster, et al., Data-driven modeling and feedback tracking control of the toroidal rotation profile for advanced tokamak scenarios in DIII-D, in: Control Applications (CCA), 2011 IEEE International Conference on, 2011, pp. 858-863. doi:10.1109/CCA.2011.6044371.

[37] H. Khalil, Nonlinear Systems, 3rd Edition, Prentice Hall, New Jersey, 2001. 